\title{
Charged Lepton Flavor Violation and the new Mu2e and COMET experiments
}

\section{Bertrand Echenard - Caltech}

APS meeting - April 2018 - Columbus 


\section{Introduction}

Charged lepton flavor violating (CLFV) processes are reactions involving $e, \mu$ and $\tau$ lepton states that don't conserve lepton family number(s).

- CLF conserving reaction: $\mu \rightarrow e v_{\mu} \bar{v}_{e}$

- CLF violating reaction: $\mu \rightarrow e$

$$
\begin{aligned}
& \tau \rightarrow \mu \mu \mu \\
& K_{L} \rightarrow \mu e \\
& H \rightarrow \tau \mu
\end{aligned}
$$




\section{Introduction}

Charged lepton flavor violating (CLFV) processes are reactions involving $e, \mu$ and $\tau$ lepton states that don't conserve lepton family number(s).

- CLF conserving reaction: $\mu \rightarrow e v_{\mu} \bar{v}_{e}$

- CLF violating reaction: $\mu \rightarrow e$

$$
\begin{aligned}
& \tau \rightarrow \mu \mu \mu \\
& K_{L} \rightarrow \mu e \\
& H \rightarrow \tau \mu
\end{aligned}
$$

CLFV are very sensitive and generic probes of new physics, exploring the underlying symmetries, and more generally the physics of flavor and families

Complementary to other searches at low- and high-energies

Exciting new initiatives searching for mu-e conversion: COMET and Mu2e 


\section{Family number: not a fundamental symmetry}

Family number is not a fundamental symmetry of the SM.

We know that quark family number is violated in weak decays

mixing strength encoded in CKM matrix

We observed neutrino mixing, which implies neutral lepton flavor violation, and evidence for BSM physics

mixing parameters encoded in PMNS matrix

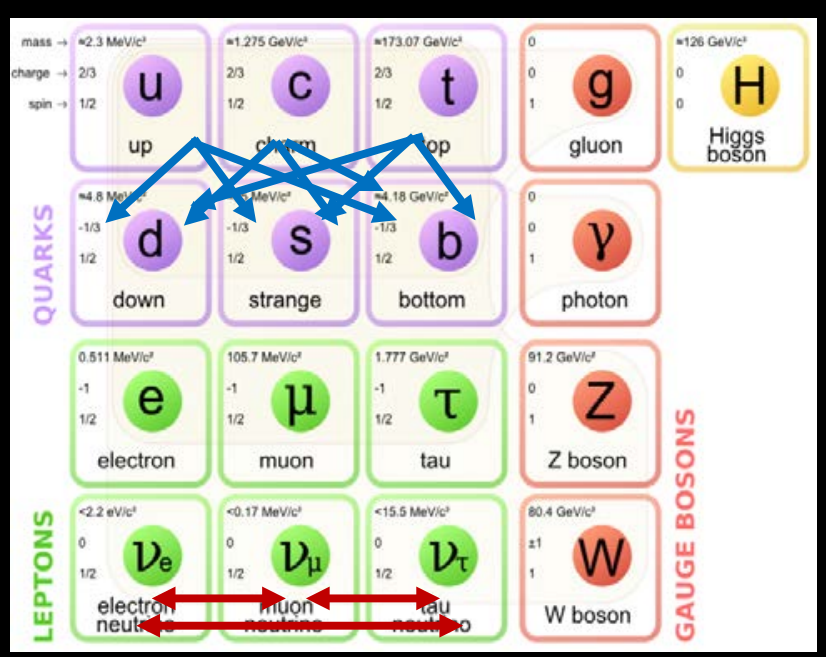

What about charged leptons?

Strictly speaking, CLFV is forbidden in the SM, since charged lepton

number is accidentally conserved when neutrinos are massless.

But... 


\section{CLFV and BSM physics}

CLFV can be generated at least at loop level with massive neutrinos:

these processes are extremely suppressed in the SM, due to GIM mechanism and tiny neutrino masses. For example:

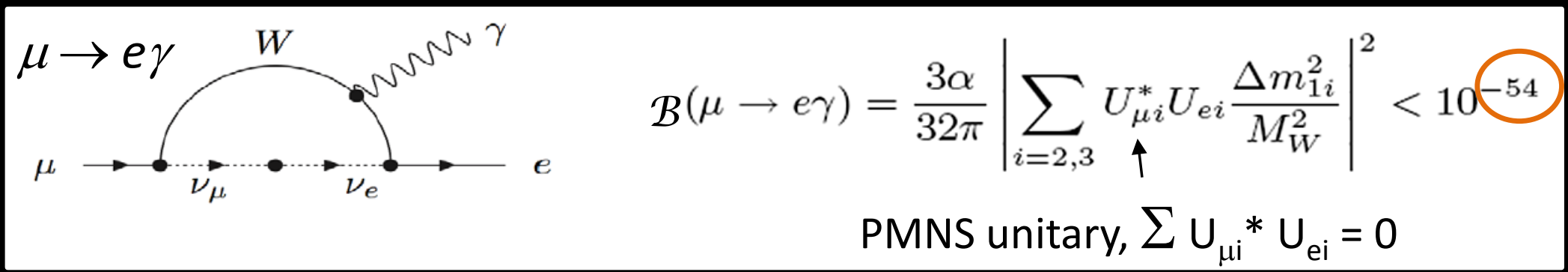

Effectively zero in the SM - background free !!!

By comparison, this mechanism is much less efficient in the quark sector

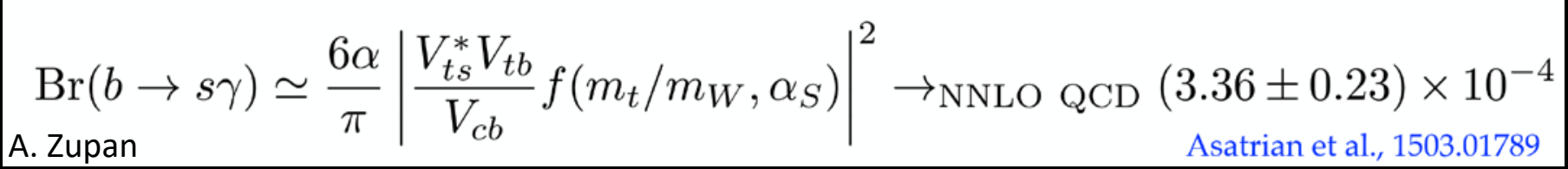

\section{Observation of CLFV is an unambiguous sign of BSM physics}


Many BSM scenarios predict observable CLFV rates.
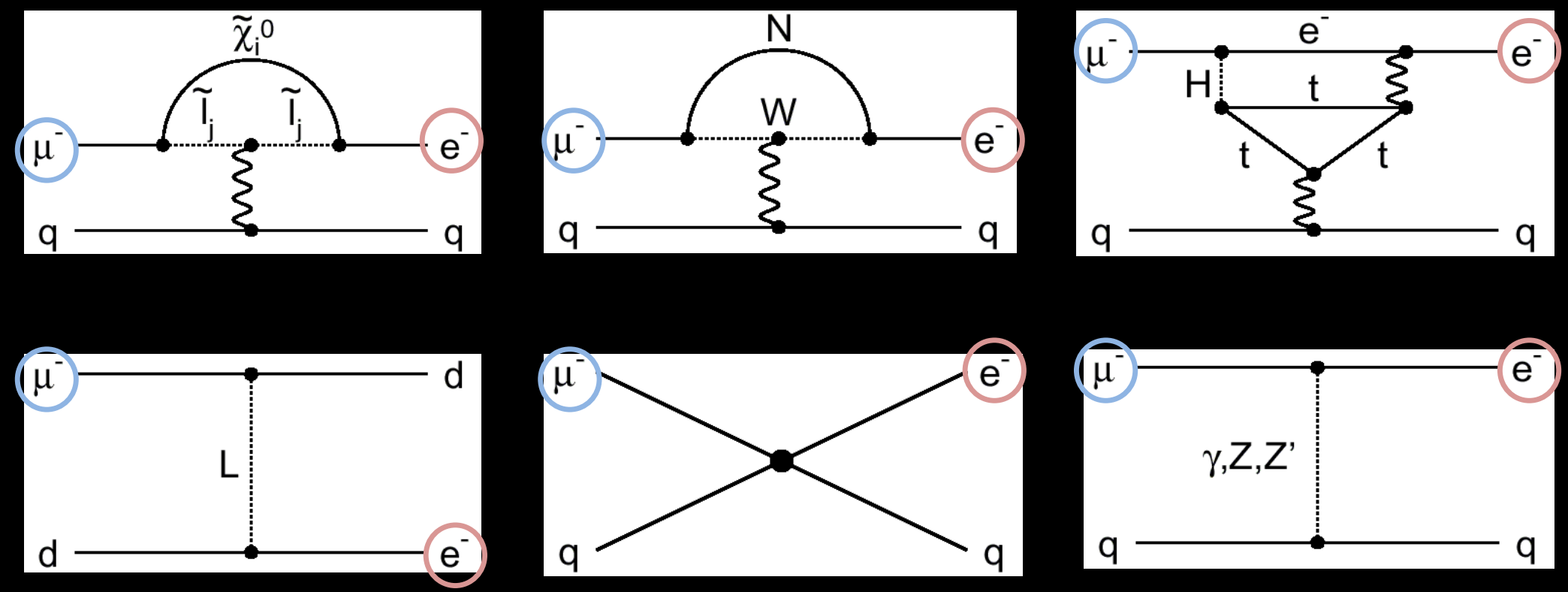

A measurement only provide indirect information, you don't directly observe the new particle(s).

Need multiple measurements to pinpoint the BSM physics and extract the CLFV couplings (the Yukawas) 


\section{Model discrimination}

\section{SUSY GUT}

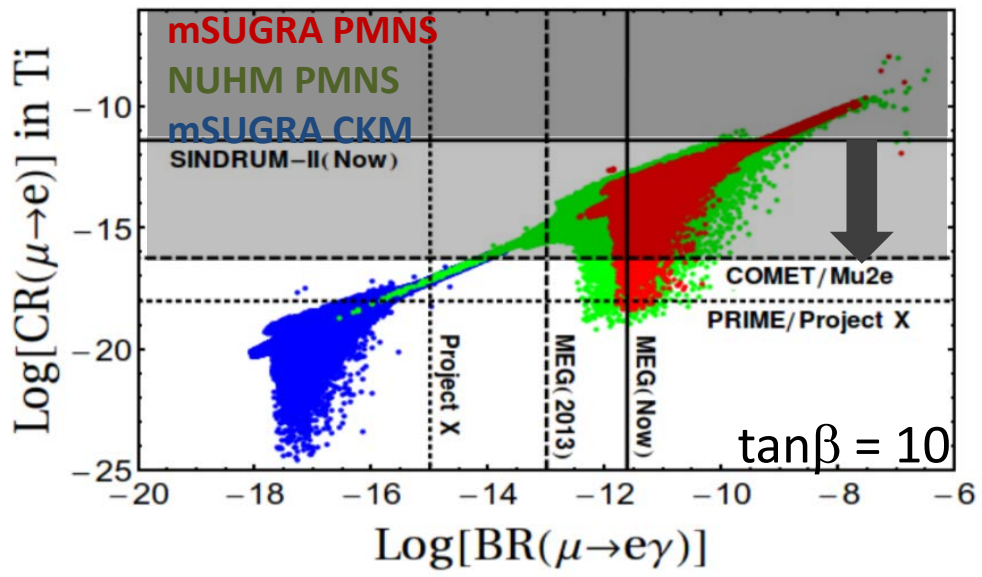

L. Calibbi et al., JHEP 1211 (2012) 040

\section{Sterile neutrino model}

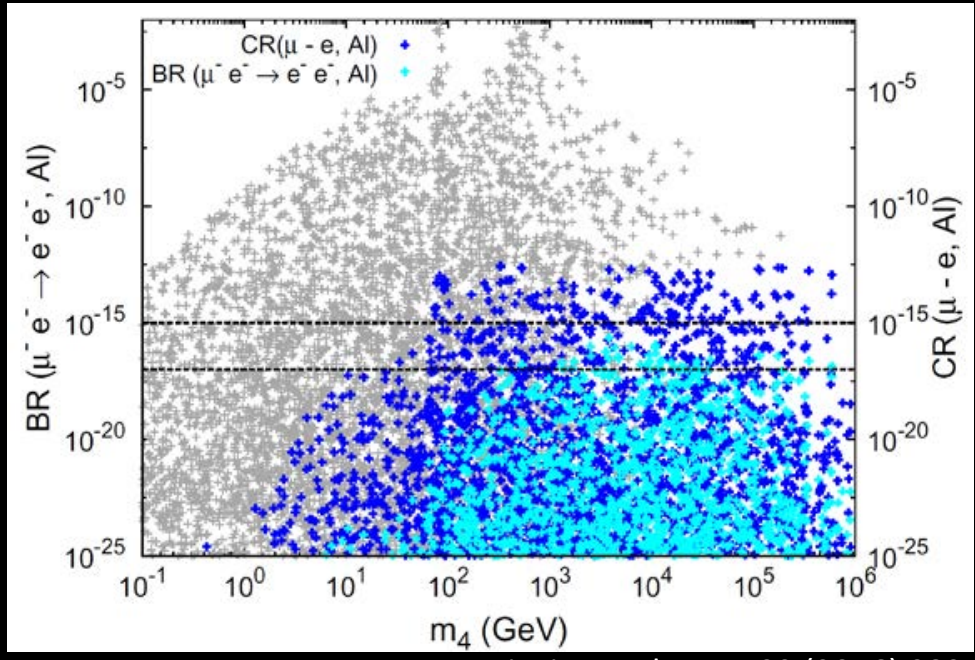

Teixeira et al., JHEP02 (2016) 083

\section{LITTLEST HIGGS}

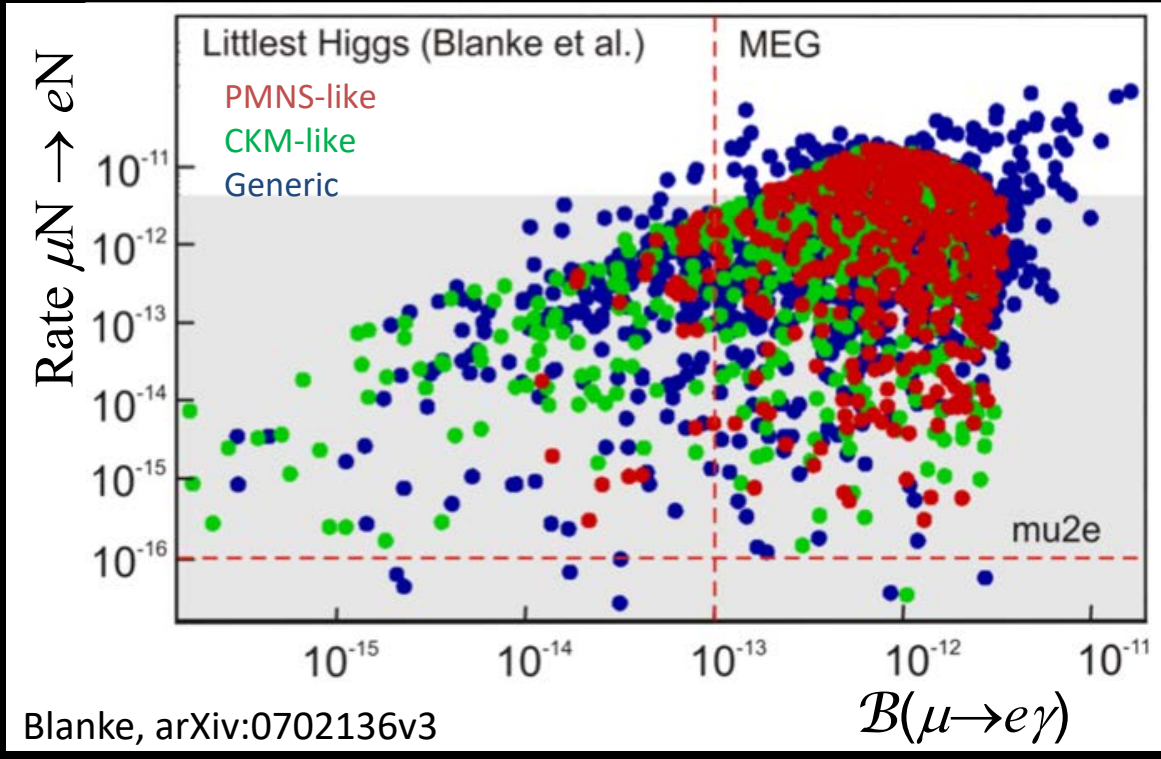

\begin{tabular}{|c|c|c|c|c|}
\hline ratio & LHT & MSSM (dipole) & MSSM (Higgs) & SM4 \\
\hline \hline$\frac{\operatorname{Br}\left(\mu^{-} \rightarrow e^{-} e^{+} e^{-}\right)}{\operatorname{Br}(\mu \rightarrow e \gamma)}$ & $0.02 \ldots 1$ & $\sim 6 \cdot 10^{-3}$ & $\sim 6 \cdot 10^{-3}$ & $0.06 \ldots 2.2$ \\
$\frac{\operatorname{Br}\left(\tau^{-} \rightarrow e^{-} e^{+} e^{-}\right)}{\operatorname{Br}(\tau \rightarrow e \gamma)}$ & $0.04 \ldots 0.4$ & $\sim 1 \cdot 10^{-2}$ & $\sim 1 \cdot 10^{-2}$ & $0.07 \ldots 2.2$ \\
$\frac{\operatorname{Br}\left(\tau^{-} \rightarrow \mu^{-} \mu^{+} \mu^{-}\right)}{\operatorname{Br}(\tau \rightarrow \mu \gamma)}$ & $0.04 \ldots 0.4$ & $\sim 2 \cdot 10^{-3}$ & $0.06 \ldots 0.1$ & $0.06 \ldots 2.2$ \\
\hline$\frac{\operatorname{Br}\left(\tau^{-} \rightarrow e^{-} \mu^{+} \mu^{-}\right)}{\operatorname{Br}(\tau \rightarrow e \gamma)}$ & $0.04 \ldots 0.3$ & $\sim 2 \cdot 10^{-3}$ & $0.02 \ldots 0.04$ & $0.03 \ldots 1.3$ \\
$\frac{\operatorname{Br}\left(\tau^{-} \rightarrow \mu^{-} e^{+} e^{-}\right)}{\operatorname{Br}(\tau \rightarrow \mu \gamma)}$ & $0.04 \ldots 0.3$ & $\sim 1 \cdot 10^{-2}$ & $\sim 1 \cdot 10^{-2}$ & $0.04 \ldots 1.4$ \\
$\frac{\operatorname{Br}\left(\tau^{-} \rightarrow e^{-} e^{+} e^{-}\right)}{\operatorname{Br}\left(\tau^{-} \rightarrow e^{-} \mu^{+} \mu^{-}\right)}$ & $0.8 \ldots 2$ & $\sim 5$ & $0.3 \ldots 0.5$ & $1.5 \ldots 2.3$ \\
$\frac{\operatorname{Br}\left(\tau^{-} \rightarrow \mu^{-} \mu^{+} \mu^{-}\right)}{\operatorname{Br}\left(\tau^{-} \rightarrow \mu^{-} e^{+} e^{-}\right)}$ & $0.7 \ldots 1.6$ & $\sim 0.2$ & $5 \ldots 10$ & $1.4 \ldots 1.7$ \\
\hline$\frac{\operatorname{R}(\mu \mathrm{Ti} \rightarrow e T \mathrm{~T})}{\operatorname{Br}(\mu \rightarrow e \gamma)}$ & $10^{-3} \ldots 10^{2}$ & $\sim 5 \cdot 10^{-3}$ & $0.08 \ldots 0.15$ & $10^{-12 \ldots 26}$ \\
\hline
\end{tabular}

Buras, Duling, Feldmann, Heidsieck, Promberger, 1006.5356 


\section{CLFV searches - a worldwide hunt}

Worldwide effort to search for experimental signatures in many different channels.
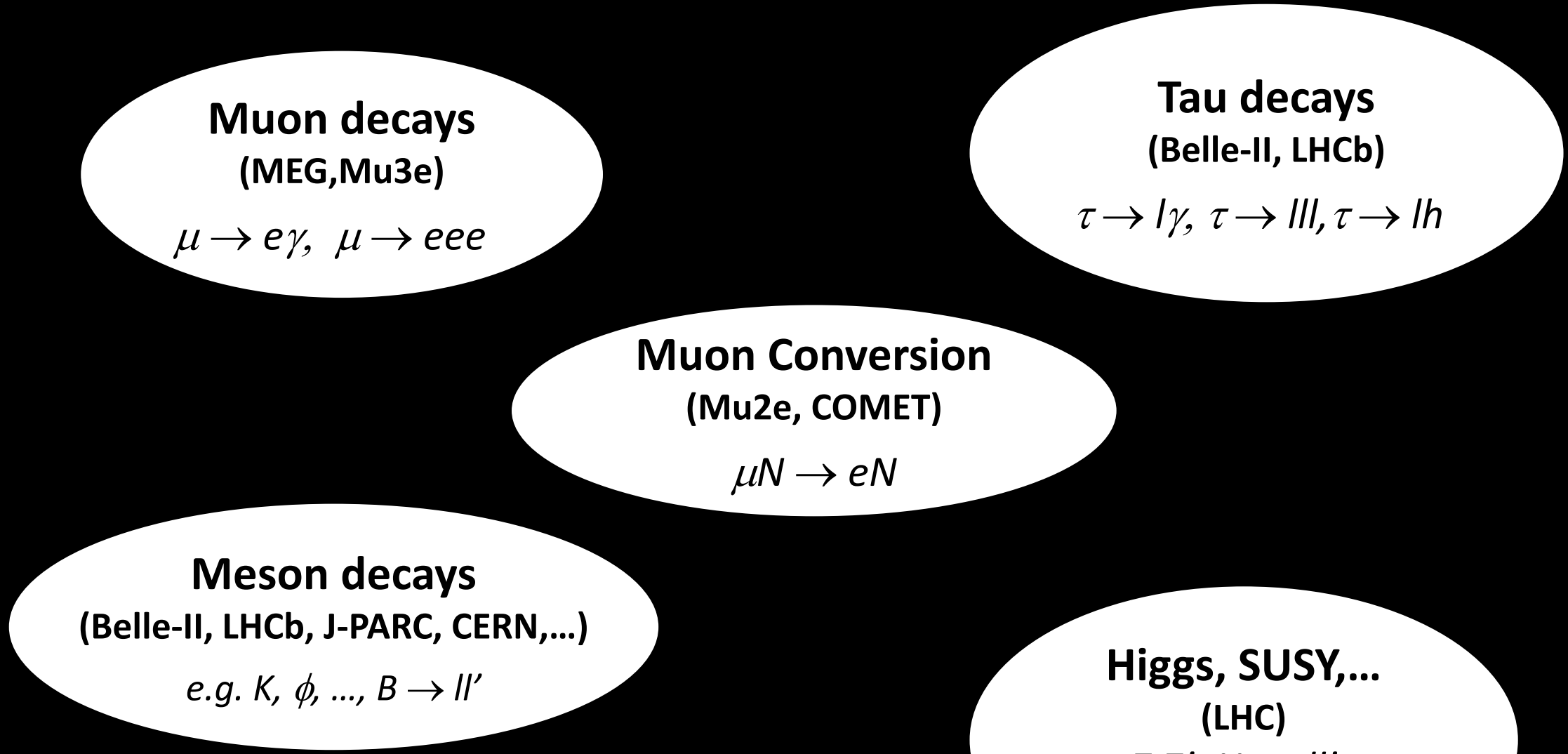

Some reviews:

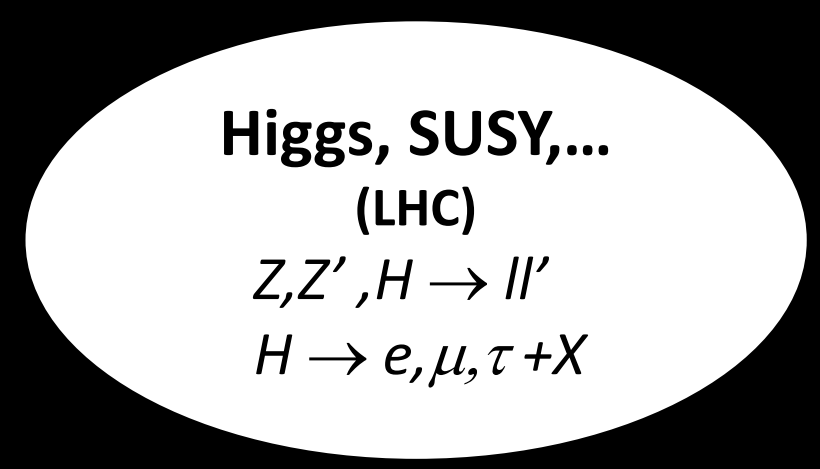

Calibi and Signorelli, arxiv: 1709.00294 Bernstein and Cooper, arxiv:1307.5787 A. de Gouvêa, P. Vogel, arXiv:1303.4097 


\section{Current and future sensitivities}

\begin{tabular}{c|l|c}
\hline Process & Current Limit & Next Generation exp \\
\hline$\tau \rightarrow e \gamma$ & $\mathrm{BR}<3.3 \times 10^{-8}$ & $10^{-9}-10^{-10}$ (Belle II) \\
$\tau \rightarrow \mu \gamma$ & $\mathrm{BR}<4.4 \times 10^{-8}$ & \\
$\tau \rightarrow \mu \mu \mu$ & $\mathrm{BR}<2.1 \times 10^{-8}$ & \\
$\tau \rightarrow e e e$ & $\mathrm{BR}<2.7 \times 10^{-8}$ & \\
\hline$K_{L} \rightarrow e \mu$ & $\mathrm{BR}<4.7 \times 10^{-12}$ & LHCb / Belle II \\
$K^{+} \rightarrow \pi^{+} e^{-} \mu^{+}$ & $\mathrm{BR}<1.3 \times 10^{-11}$ & LHCb / Belle II \\
\hline$B^{0} \rightarrow e \mu$ & $\mathrm{BR}<2.8 \times 10^{-9}$ & $10^{-14}$ (MEG) \\
$B^{+} \rightarrow K^{+} e \mu$ & $\mathrm{BR}<3.8 \times 10^{-8}$ & $10^{-16}$ (PSI) \\
\hline$\mu^{+} \rightarrow e^{+} \gamma$ & $\mathrm{BR}<4.2 \times 10^{-13}$ & $10^{-17}$ (Mu2e, COMET) \\
$\mu^{+} \rightarrow e^{+} e^{+} e^{-}$ & $\mathrm{BR}<1.0 \times 10^{-12}$ & \\
$\mu N \rightarrow e N$ & $\mathrm{R}$ & \\
\hline
\end{tabular}

New physics effects could be larger for taus than muons, but colliders produce $\mathrm{O}\left(10^{10}\right) \tau /$ year, while dedicated muon experiments can produce $\mathrm{O}\left(10^{10}\right) \mu / \mathrm{s}$.

I'll now focus on CLFV in the muon sector 


\section{Already a long history...}

There have been many searches for CLFV in muon decays

Muon an independent

lepton, no $\mu \rightarrow e \gamma$

$\mu \rightarrow e \gamma \sim 10^{-4} / 10^{-5}$ or two $v$

Feinberg (1958)

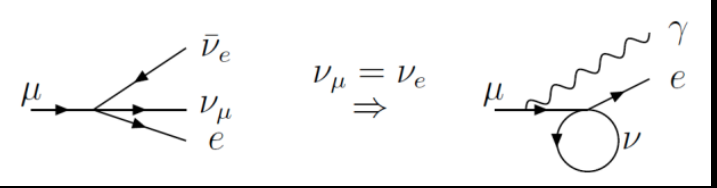

No $\mu \rightarrow e \gamma \Rightarrow$ Two neutrinos!

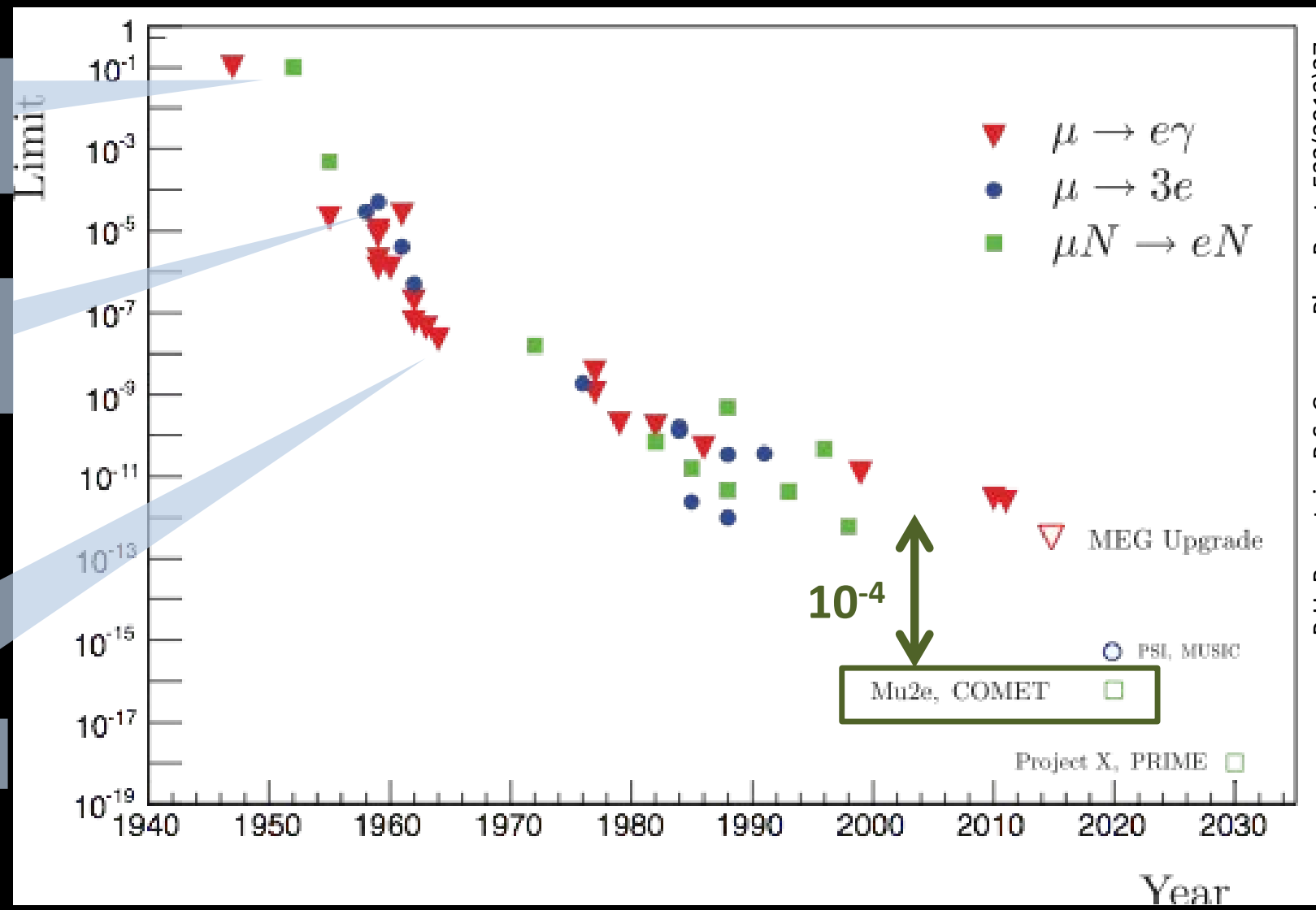

New experimental initiatives will improve upon current limits on $\mu \mathrm{N} \rightarrow e N$ conversion by four orders of magnitude! 


\section{Possible contributions to mu-e conversion}

A wide array of BSM physics induce $\mu N \rightarrow e N$ conversion, either through loops or exchange of heavy intermediate particles

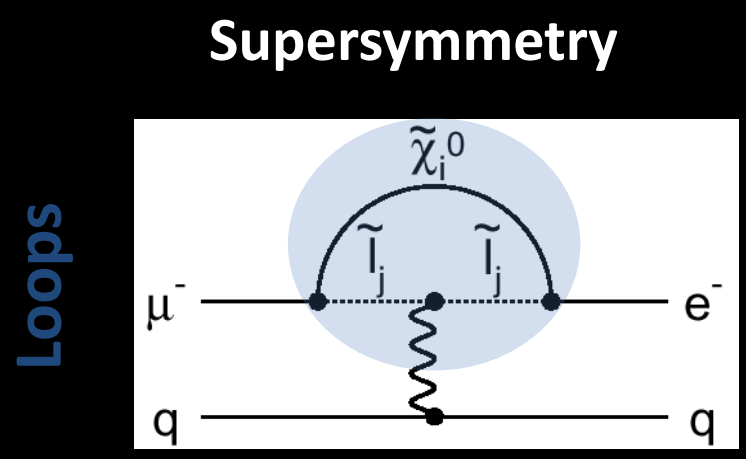

Leptoquarks

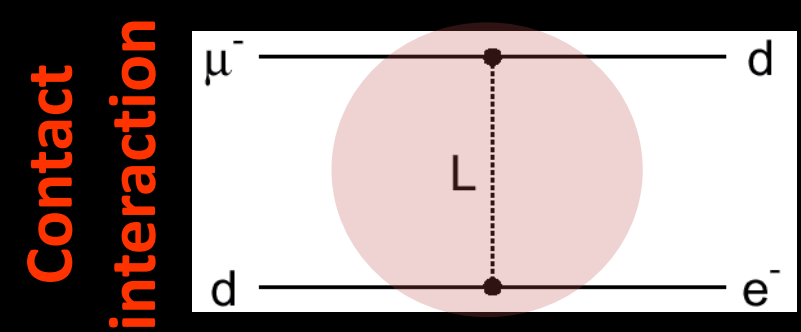

Heavy neutrino

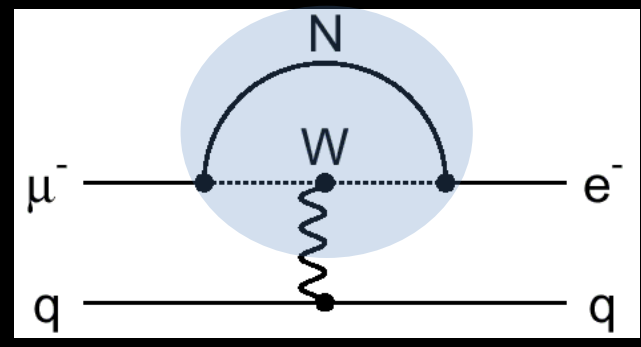

Compositness

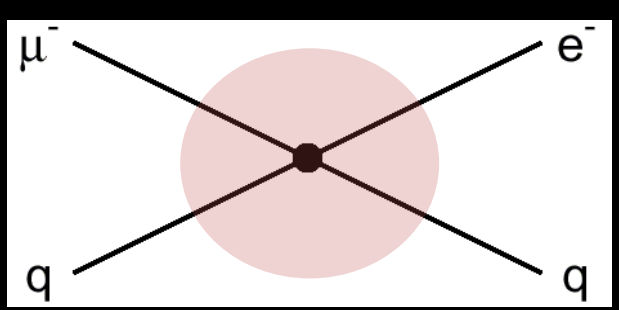

Two Higgs doublet

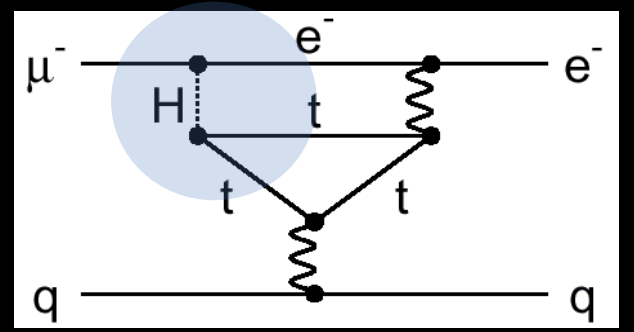

New heavy bosons / anomalous coupling

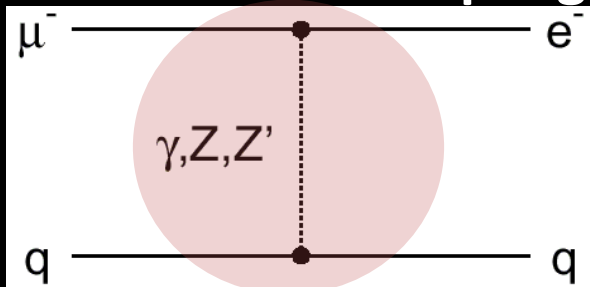




\section{Generic parametrization of CLFV process}

$$
\mathcal{L}_{\mathrm{CLFV}}=\frac{m_{\mu}}{(1+\kappa) \wedge^{2}} \bar{\mu}_{R} \sigma_{\mu \nu} e_{L} F^{\mu \nu}+\frac{\kappa}{(1+\kappa) \wedge^{2}} \bar{\mu}_{L} \gamma_{\mu} e_{L}\left(\bar{u}_{L} \gamma^{\mu} u_{L}+\bar{d}_{L} \gamma^{\mu} d_{L}\right)
$$

$\Lambda$ : effective mass scale of new physics $\kappa$ : relative contribution of the contact term

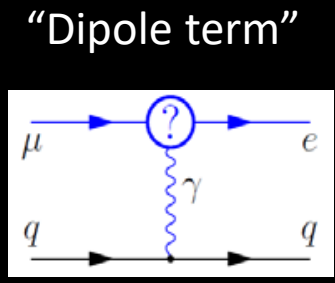

Contributes to $\mu \rightarrow e \gamma$

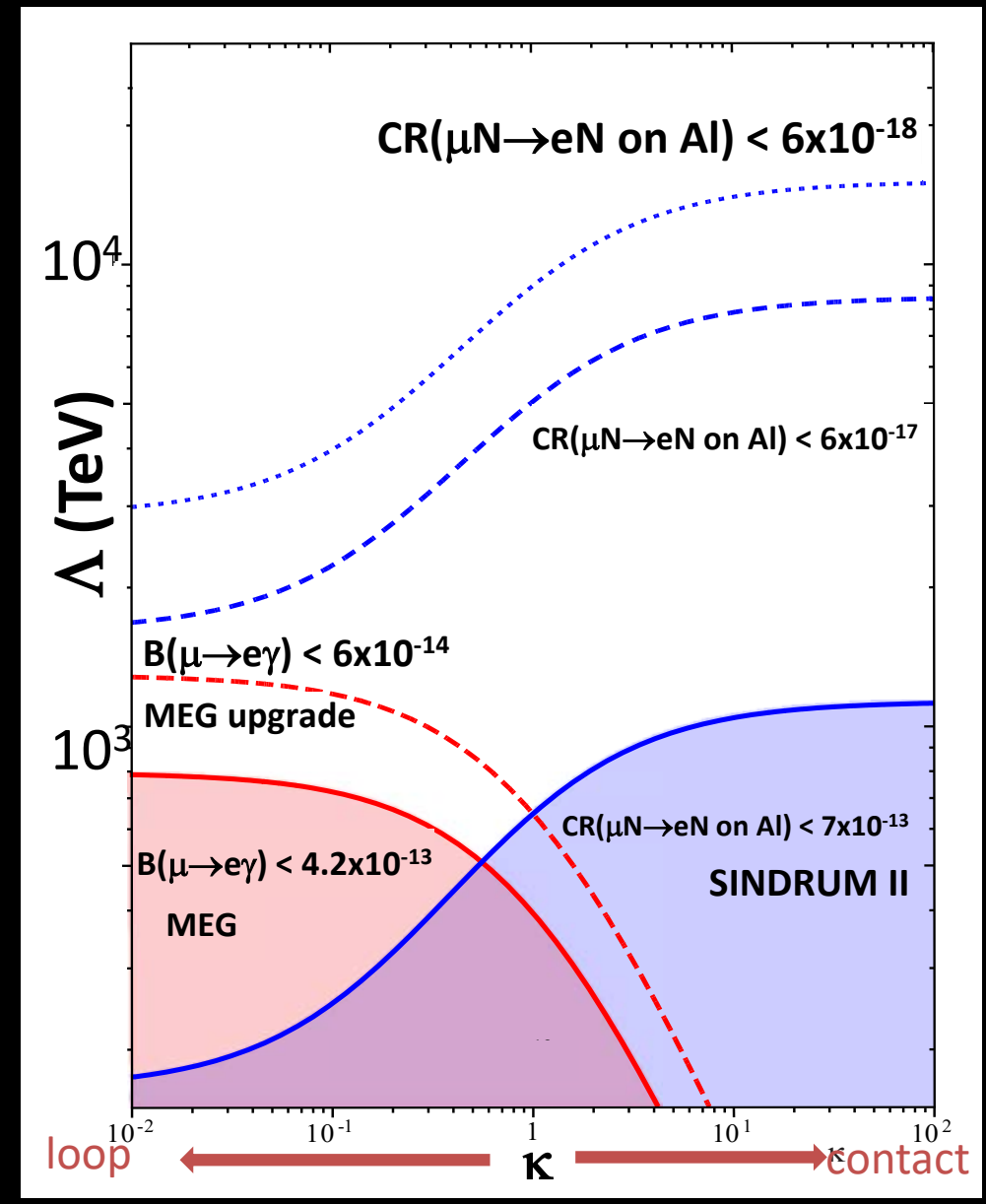

"Contact term"

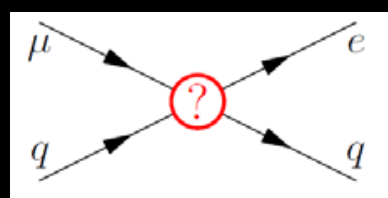

No contribution

to $\mu \rightarrow e \gamma$

CLFV can probe very high mass scales O(1000-10000 TeV)

Different BSM scenarios predict different value of $\kappa \rightarrow$ model diagnosis 


\section{MUON-TO-ELECTRON CONVERSION}

Experimental concept 


\section{Muon-to-electron conversion experiments}

\section{Search for neutrinoless muon-to-electron conversion}

Measure ratio of signal to all muon captures

Signal is mono-energetic electron and nothing else.

Electron energy is $Z$ dependent, $E_{e}=$ $105 \mathrm{MeV}$ for $\mathrm{N}=\mathrm{Al}$

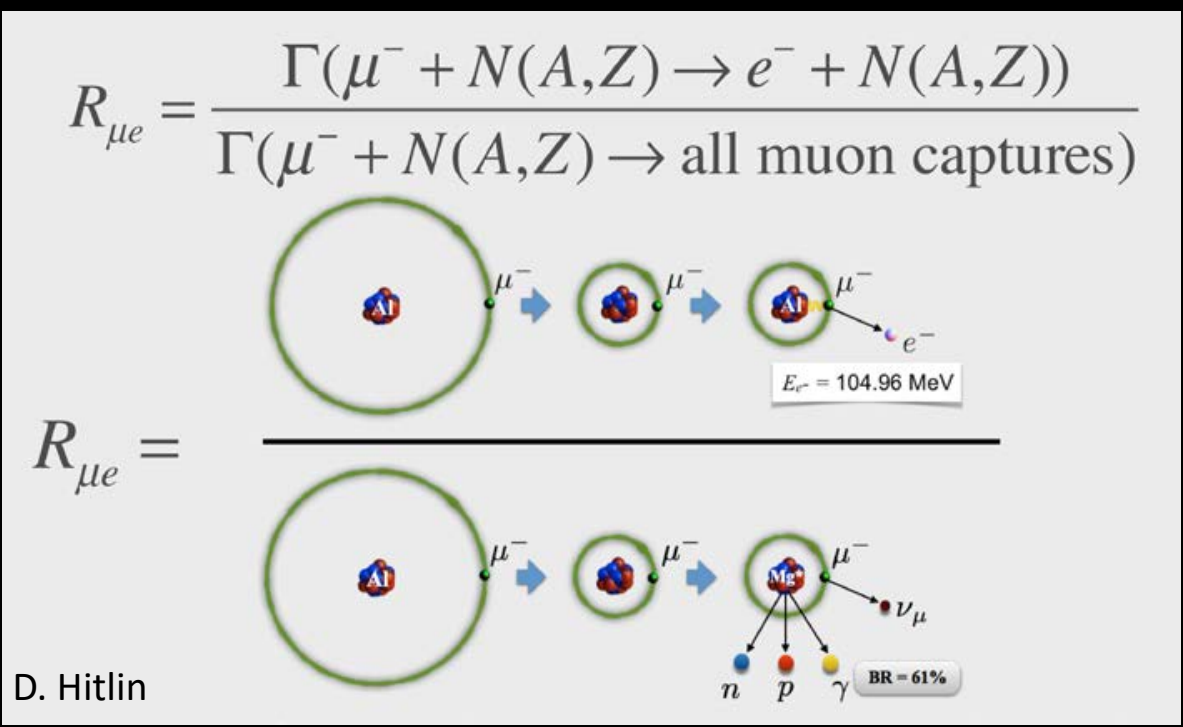

Currently four experiment in various stages of execution:

DeeMee at J-PARC COMET at J-PARC* Mu2e at FNAL*

\} Aim to achieve single event sensitivity $R_{\mu e} \sim 10^{-17}$ PRISM/Prime 


\section{Experimental concept}

\section{Experimental concept to search for muon-to-electron conversion}

- Produce beam of low-momentum muons by hitting protons on target:

$p+$ nucleus $\rightarrow \pi^{-} \rightarrow \mu^{-} v_{\mu}$

production

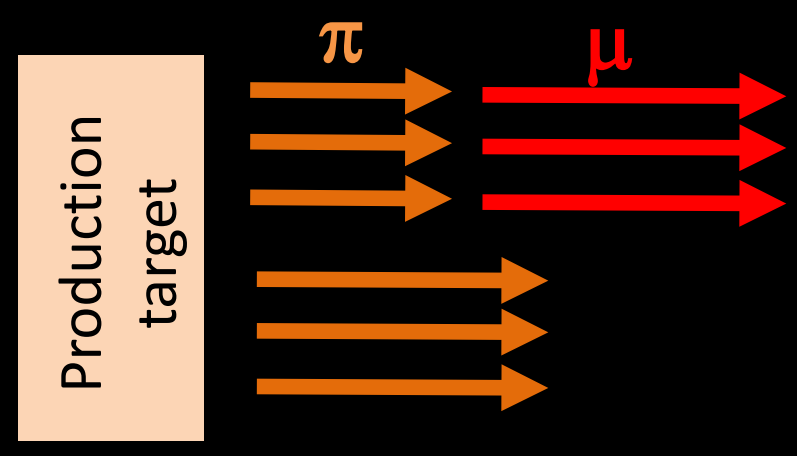




\section{Experimental concept}

\section{Experimental concept to search for muon-to-electron conversion}

- Produce beam of low-momentum muons by hitting protons on target:

$$
p+\text { nucleus } \rightarrow \pi^{-} \rightarrow \mu^{-} v_{\mu}
$$

- Collect and stop low momentum muons in stopping target

production

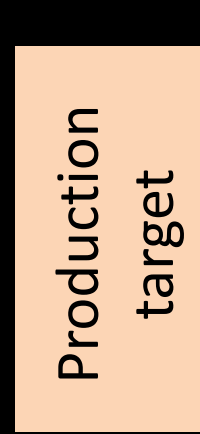

transport

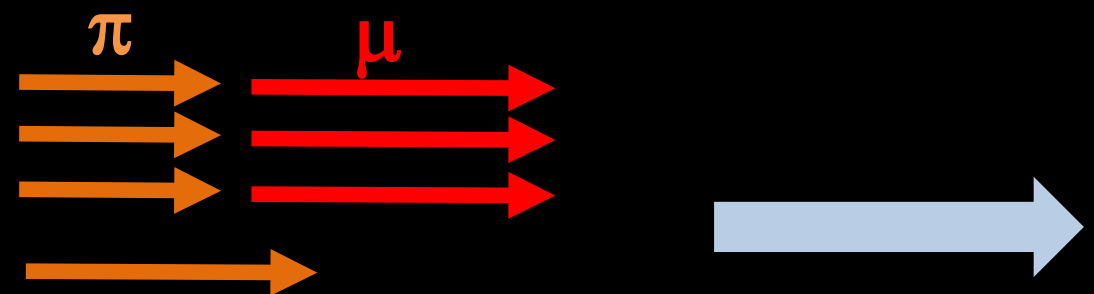

detector

\section{Stopping target}




\section{Experimental concept}

\section{Experimental concept to search for muon-to-electron conversion}

- Produce beam of low-momentum muons by hitting protons on target:

$$
p+\text { nucleus } \rightarrow \pi^{-} \rightarrow \mu^{-} v_{\mu}
$$

- Collect and stop low momentum muons in stopping target

- Muon cascade to $K$ shell $(<p s)$ firing off $X$ rays measure $X$ rays spectrum to estimate the number of captures

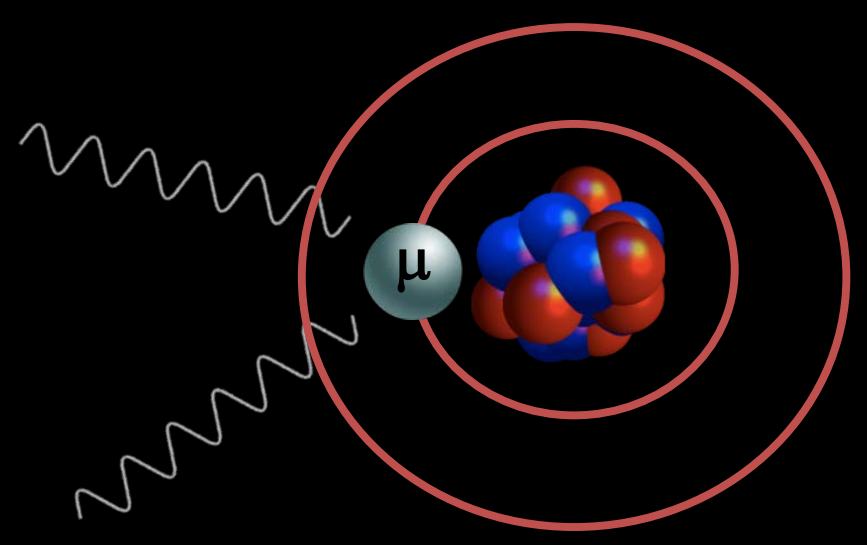




\section{Experimental concept}

\section{Experimental concept to search for muon-to-electron conversion}

- Produce beam of low-momentum muons by hitting protons on target:

$$
p+\text { nucleus } \rightarrow \pi^{-} \rightarrow \mu^{-} v_{\mu}
$$

- Collect and stop low momentum muons in stopping target

- Muon cascade to $K$ shell $(<p s)$ firing off $X$ rays measure $X$ rays spectrum to estimate the number of captures

- Wait for muon to eventually convert into an electron coherent process, the nucleus remains intact 


\section{Experimental concept}

\section{Experimental concept to search for muon-to-electron conversion}

- Produce beam of low-momentum muons by hitting protons on target:

$$
p+\text { nucleus } \rightarrow \pi^{-} \rightarrow \mu^{-} v_{\mu}
$$

- Collect and stop low momentum muons in stopping target

- Muon cascade to $K$ shell $(<p s)$ firing off $X$ rays measure $X$ rays spectrum to estimate the number of captures

- Wait for muon to eventually convert into an electron coherent process, the nucleus remains intact

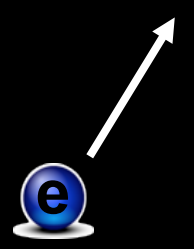

- Signal is a mono-energetic electron

$$
\begin{aligned}
E_{\mu e} & =m_{\mu} c^{2}-E_{b}-E_{\text {recoil }} \\
& =104.973 \mathrm{MeV} \quad(\text { for } \mathrm{Al})
\end{aligned}
$$

This sounds simple, but what happens most of the time... 
Nuclear capture ( $\sim 61 \%$ for $\mathrm{Al})$

$$
\mu N \rightarrow v_{\mu} N^{*}
$$

Muon decay in orbit ( $39 \%$ for Al)

$$
\mu \rightarrow e v_{\mu} \bar{v}_{e}
$$

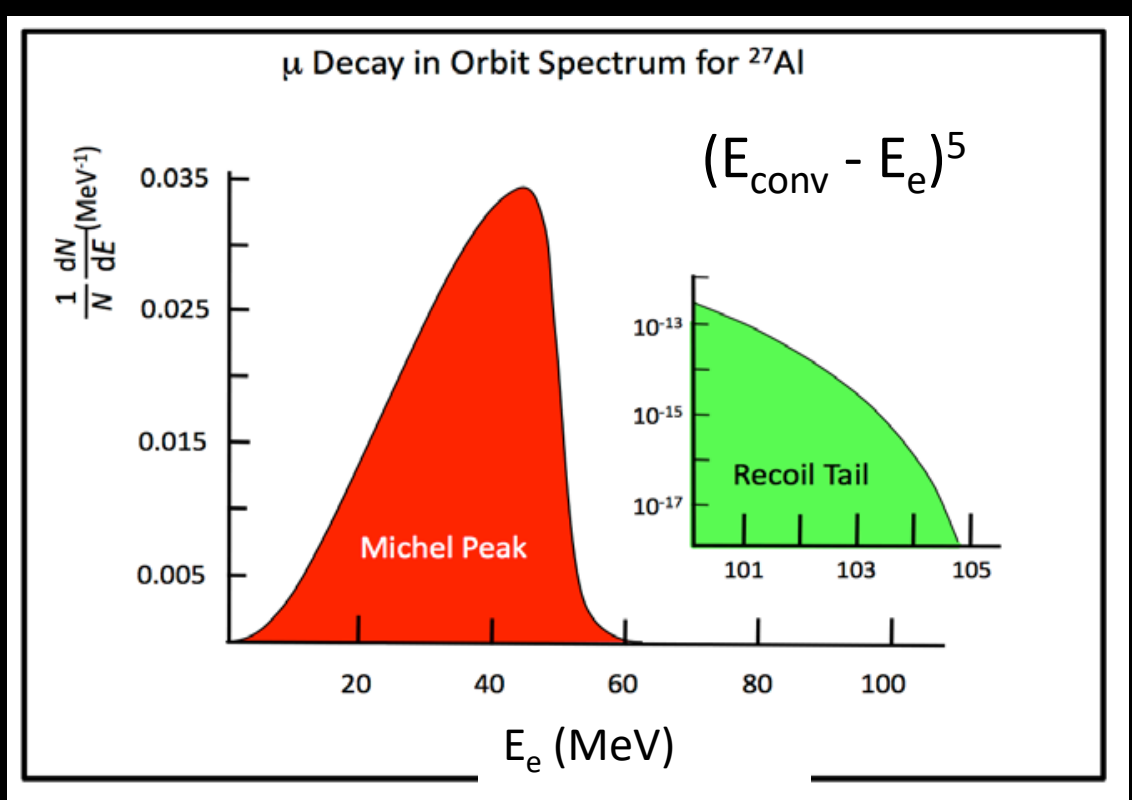

See e.g. Czarnecki et al., Phys. Rev. D 84, 013006 (2011)

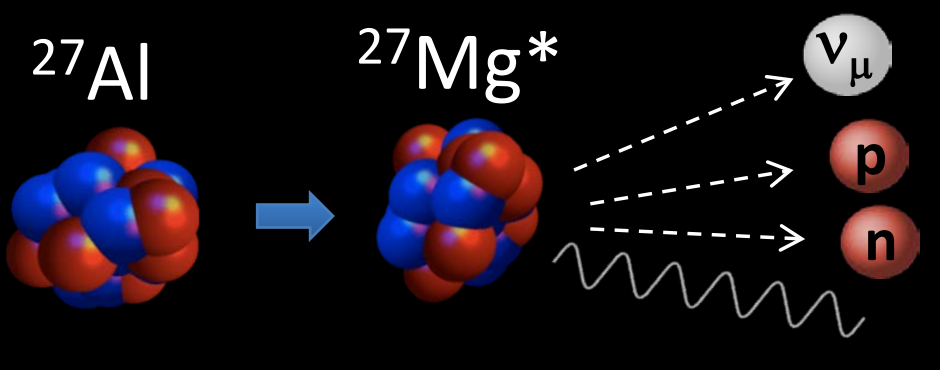

Could produce signal-like electron

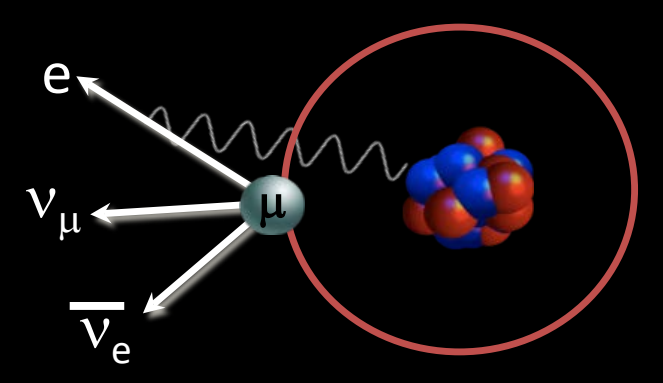

The Michel spectrum is distorted by the presence of the nucleus and the electron can be at the conversion energy if the neutrinos are almost at rest 


\section{And other backgrounds...}

\section{Prompt background}

Particles produced in addition to the muons by primary protons which interact almost immediately when they hit the stopping target: pions, neutrons, antiprotons.

- Radiative pion capture (RPC)

$$
\begin{aligned}
& \pi^{-} N \rightarrow \gamma N^{\prime}, \gamma \rightarrow e^{+} e^{-} \\
& \pi^{-} N \rightarrow e^{+} e^{-} N^{\prime}
\end{aligned}
$$

- Pion/muon decays in flight

\section{Other background}

- Antiprotons producing pions when annihilating in the target

- Cosmic rays induced,...

Need to control the backgrounds extremely well !!! 


\section{Pulse muon beam}

Pulse muon beam with a long interval between bunches to suppress prompt background

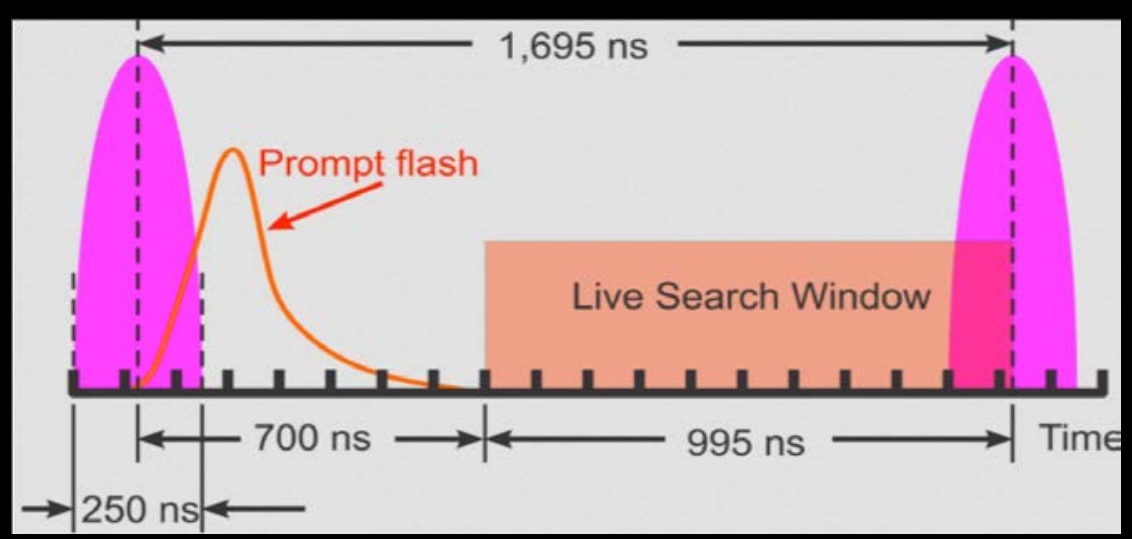

Prompt background decreases rapidly and becomes negligible in the search window must suppress out-of-time proton by $\mathrm{O}\left(10^{10}\right)$

Muonic atom lifetime large enough to have good signal efficiency

\section{Capture solenoids*}

Place production target inside magnetic field to trap more pions

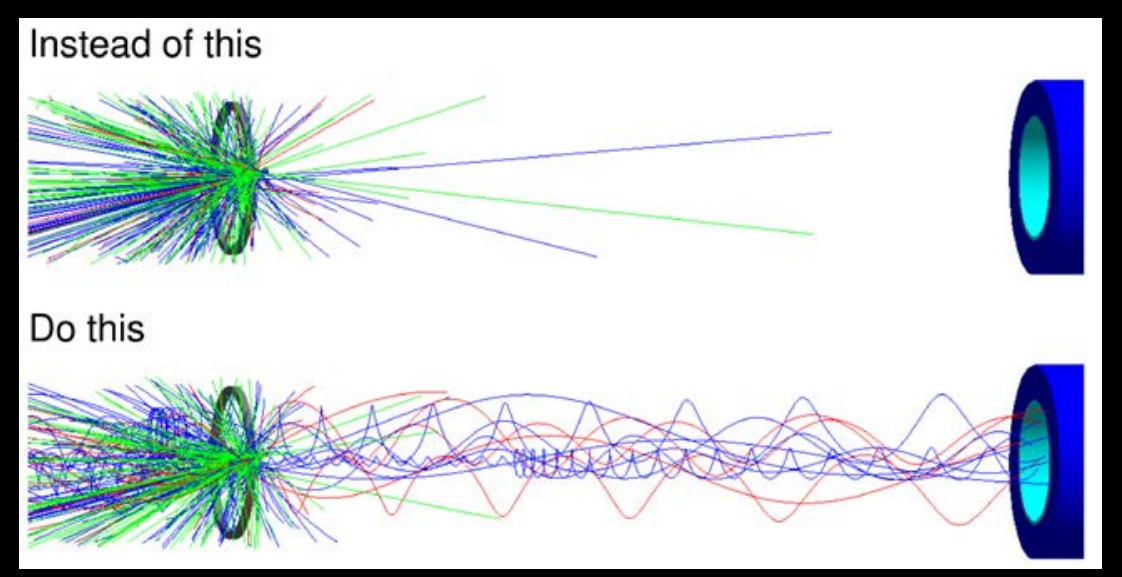

Large number of muons with reasonable beam current 
MUON-TO-ELECTRON CONVERSION

New experiments 


\section{New experimental efforts}

Mu2e at FNAL
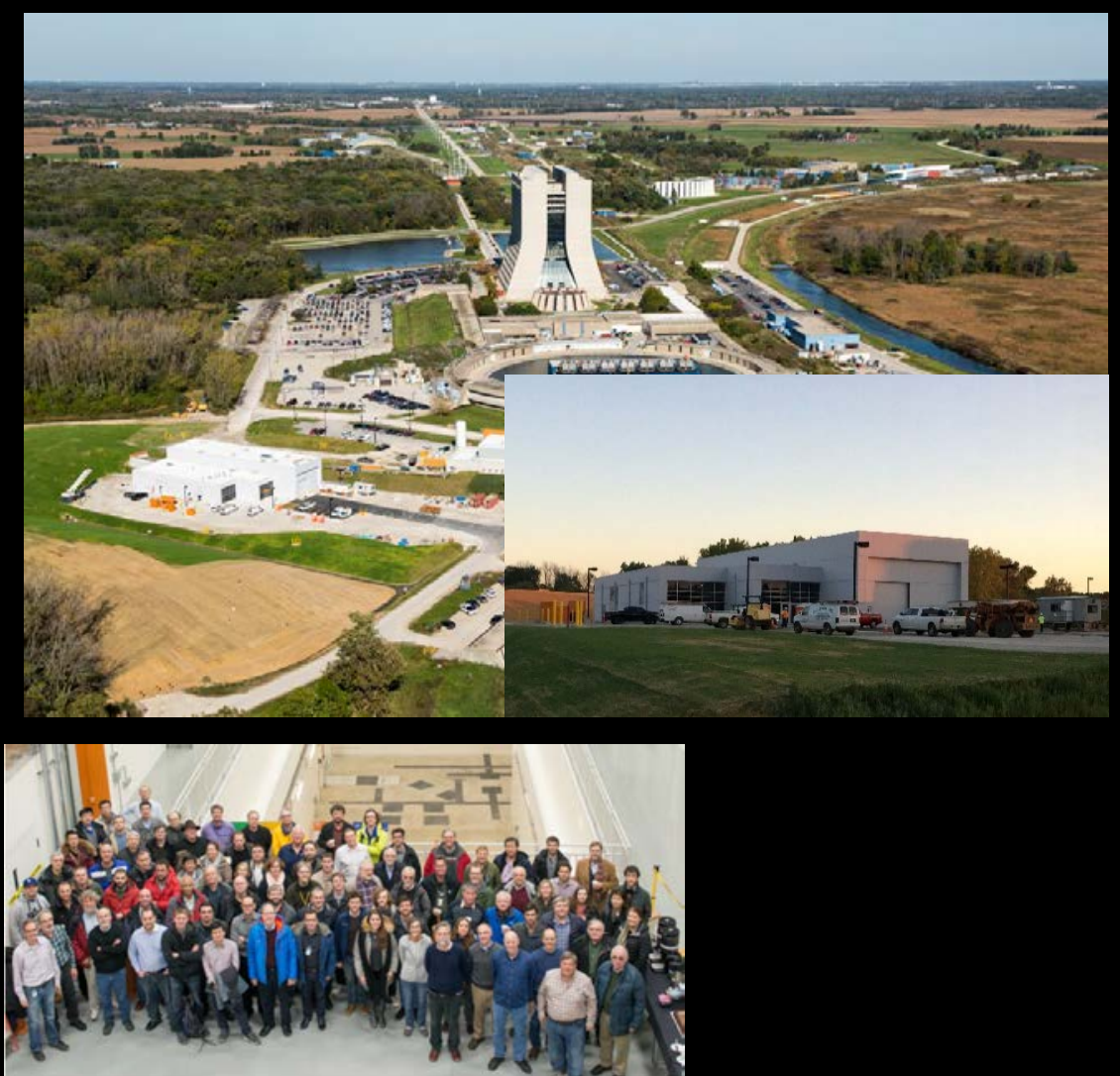

Over 200 scientists

37 institutions,

5 countries

\section{COMET at JPARC}

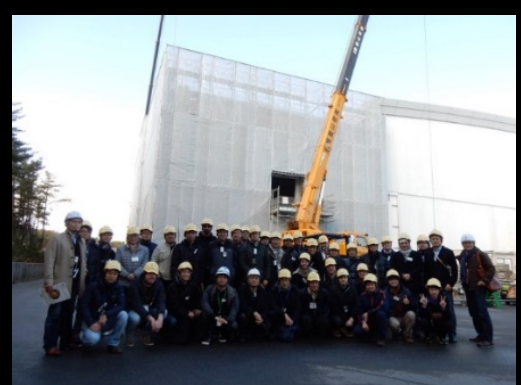

Over 150 collaborators

33 institutes,

15 countries

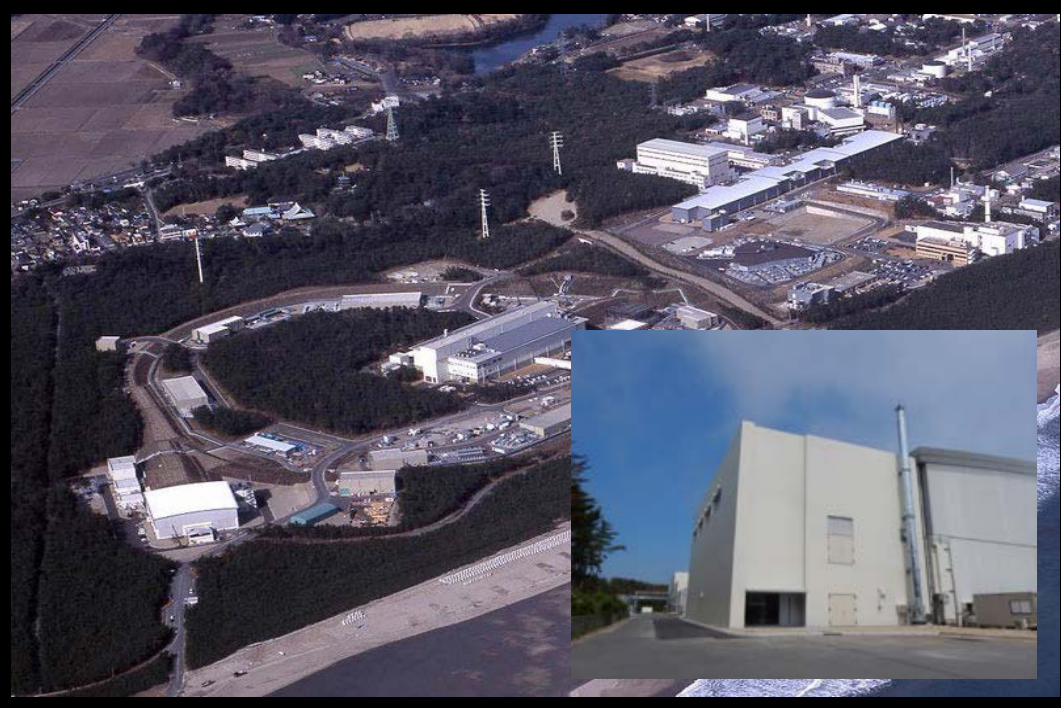




\section{Mu2e experiment - overview}

\section{Production Target / Solenoid (PS)}

- Proton beam strikes target, producing mostly pions

- Graded magnetic field contains pions/muons and collimate them into transport solenoid $\rightarrow$ high muon intensity

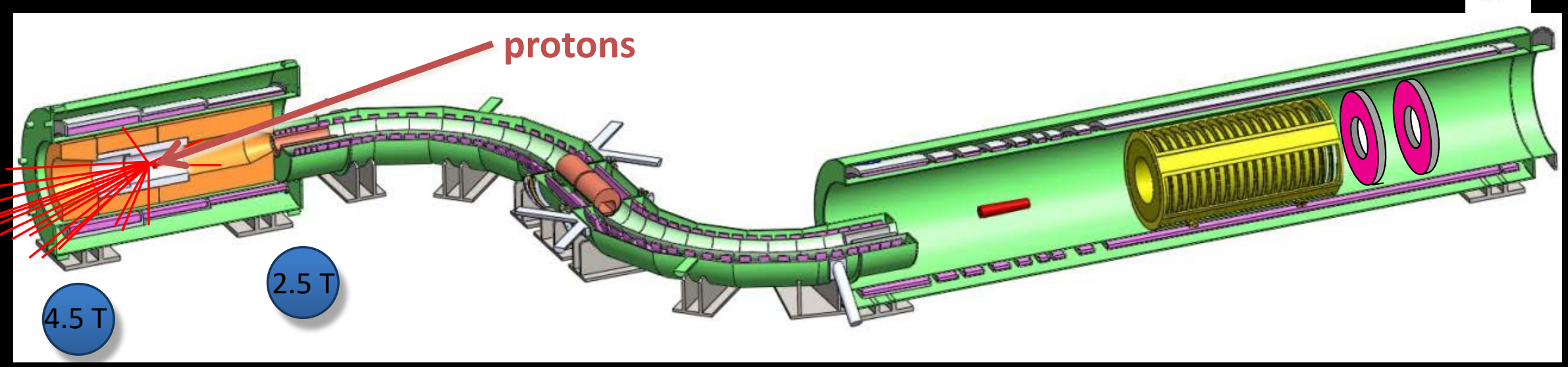




\section{Mu2e experiment - overview}

\section{Production Target / Solenoid (PS)}

- Proton beam strikes target, producing mostly pions

- Graded magnetic field contains pions/muons and collimate them into transport solenoid $\rightarrow$ high muon intensity

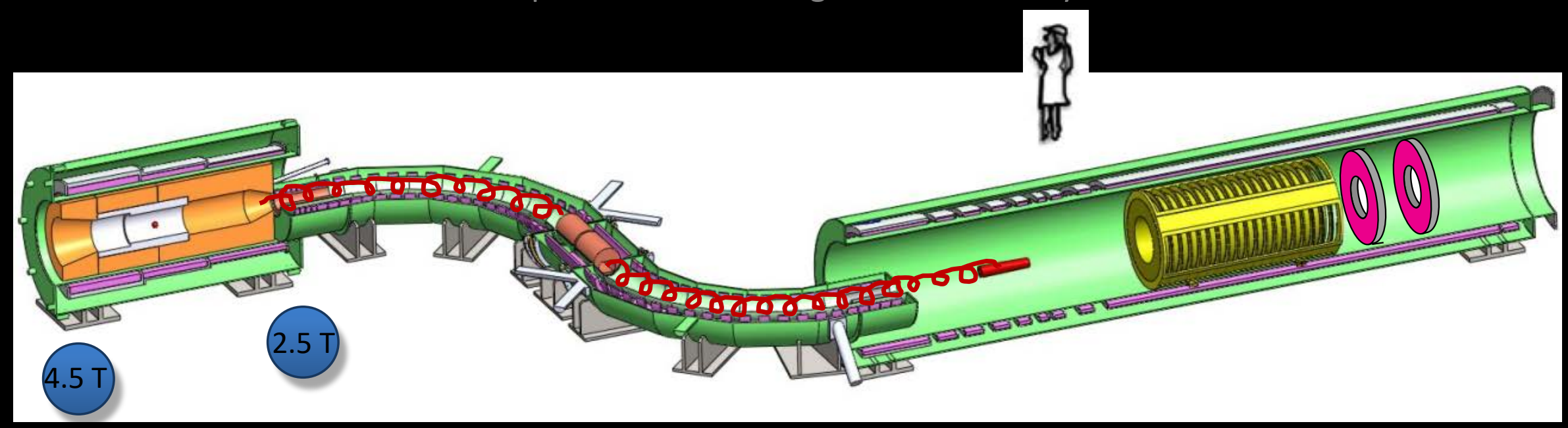

\section{Transport Solenoid (TS)}

- Collimator selects low momentum, negative muons

- Antiproton absorber

- The S shape eliminates photons and neutrons 


\section{Mu2e experiment - overview}

\section{Production Target / Solenoid (PS)}

- Proton beam strikes target, producing mostly pions

- Graded magnetic field contains pions/muons and collimate them into transport solenoid $\rightarrow$ high muon intensity

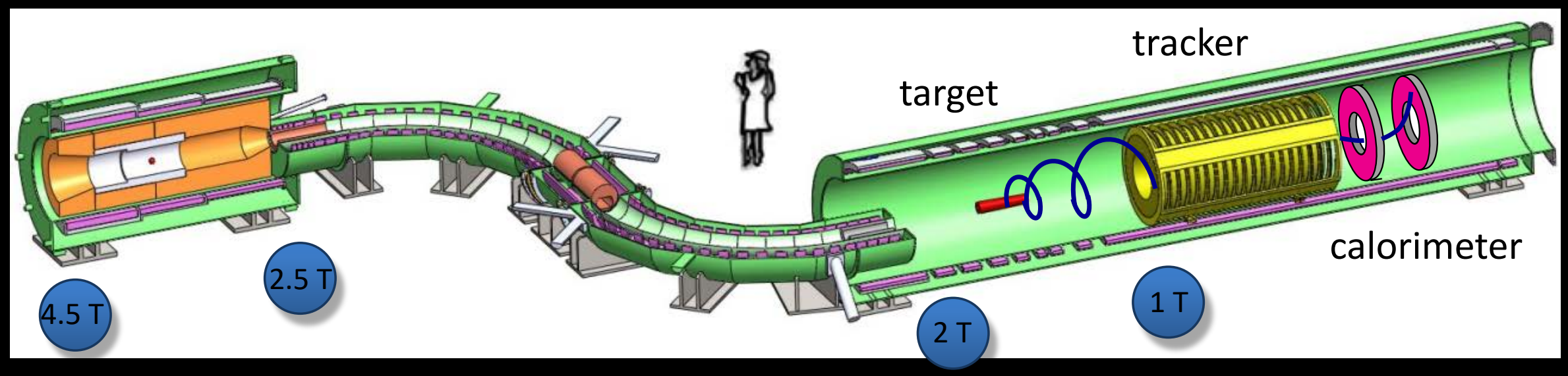

\section{Transport Solenoid (TS)}

- Collimator selects low momentum, negative muons

- Antiproton absorber

- The S shape eliminates photons and neutrons

\section{Target, Detector and Solenoid (DS)}

- Capture muons on Al target

- Measure momentum in tracker and energy in calorimeter

- Graded field "reflects" conversion electrons, improving efficiency 


\section{Mu2e experiment - overview}

\section{Production Target / Solenoid (PS)}

- Proton beam strikes target, producing mostly pions

- Graded magnetic field contains pions/muons and collimate them into transport solenoid $\rightarrow$ high muon intensity

\section{REPEAT}

- next proton bunch 1695 ns later

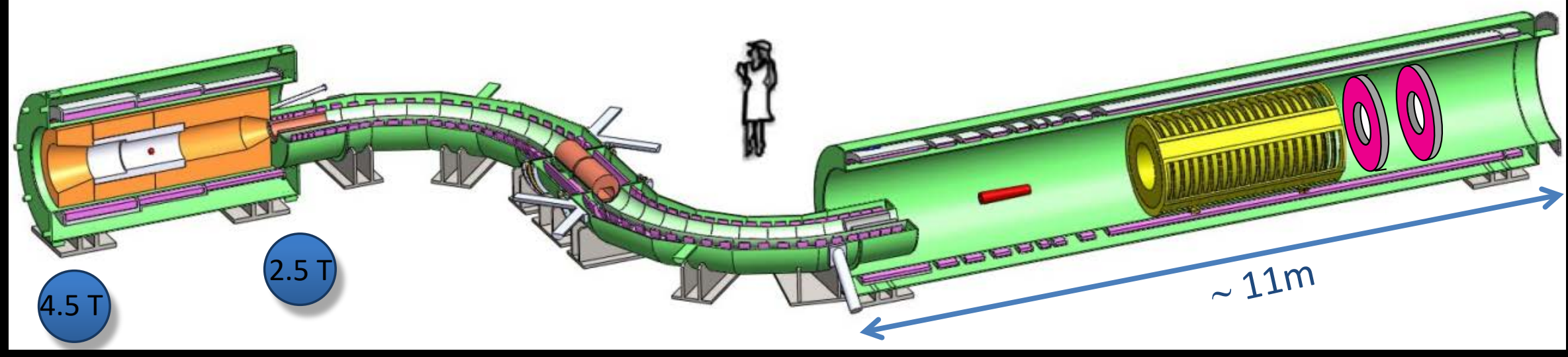

\section{Transport Solenoid (TS)}

- Collimator selects low momentum, negative muons

- Antiproton absorber

- The S shape eliminates photons and neutrons

\section{Target, Detector and Solenoid (DS)}

- Capture muons on Al target

- Measure momentum in tracker and energy in calorimeter

- Graded field "reflects" conversion electrons, improving efficiency 


\section{Mu2e experiment - tracker}

The tracker is designed to measure the momentum of charged particles with high precision and ensure operability in a high background environment.
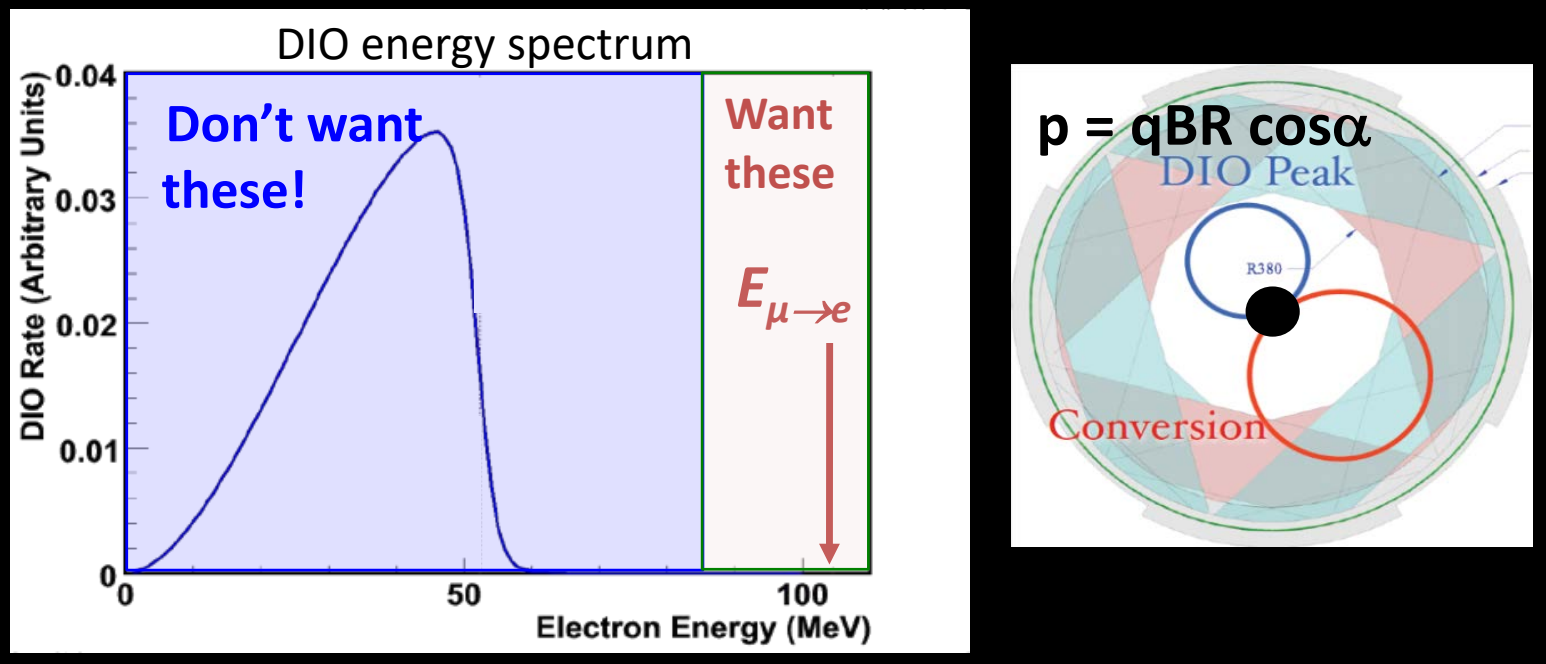

Since track radius is proportional to energy, design the tracker to only detect tracks with large enough radii.

Low-mass, annular tracker made of straw panels

Excellent rate capabilities and resolution $\left(\sigma_{\mathrm{p}} / \mathrm{p}<180\right.$ $\mathrm{keV} / \mathrm{c}$ at $105 \mathrm{MeV} / \mathrm{c}$ )

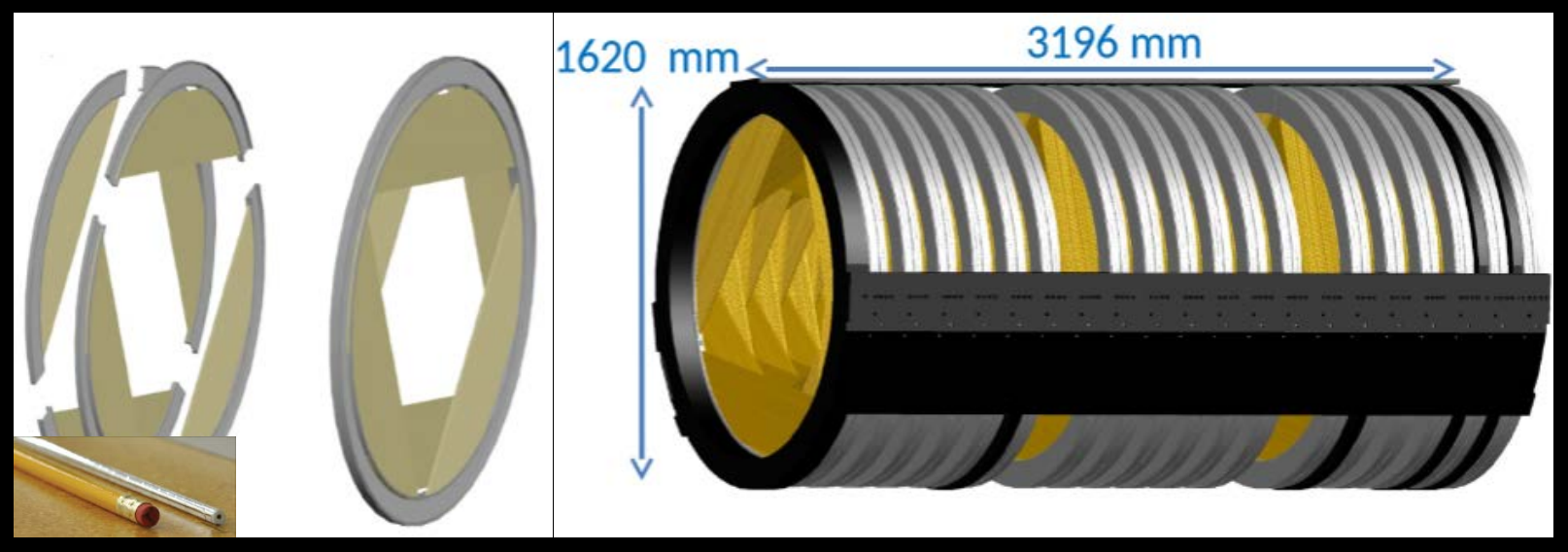




\section{Mu2e experiment - expected sensitivity}

3 years running period with $1.2 \times 10^{20}$ protons on target per year

\section{Estimated background}

\begin{tabular}{|l|l|}
\hline Process & Expected event yield \\
\hline Cosmic rays & $0.209 \pm 0.022$ (stat) \pm 0.055 (syst) \\
DIO & $0.144 \pm 0.028$ (stat) \pm 0.11 (syst) \\
Antiprotons & $0.040 \pm 0.001$ (stat) \pm 0.020 (syst) \\
Pion capture & $0.021 \pm 0.001$ (stat) \pm 0.002 (syst) \\
Muon DIF & $<0.003$ \\
Pion DIF & $0.001 \pm<0.001$ \\
Beam electrons & $(2.1 \pm 1.0) \times 10^{-4}$ \\
RMC & $0.000_{-0.000}^{+0.004}$ \\
\hline Total & $0.41 \pm 0.13$ (stat+syst) \\
\hline
\end{tabular}

\section{Reconstructed $\mathrm{e}^{-}$momentum}

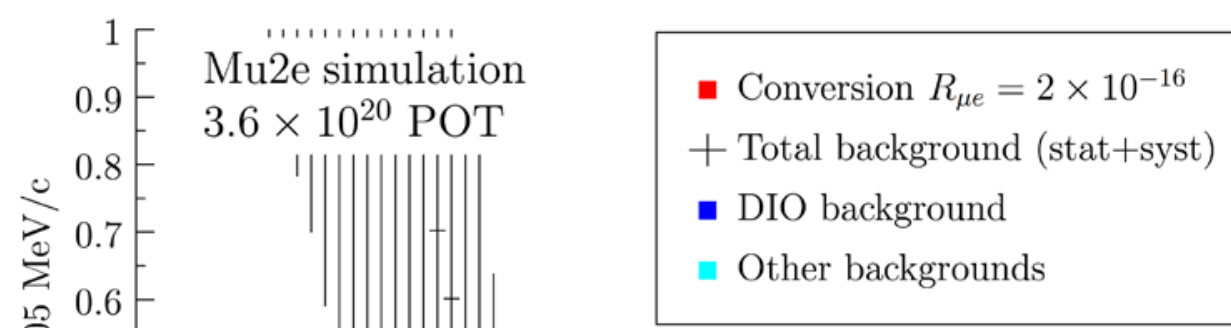

+ Total background (stat+syst)

- DIO background

Other backgrounds

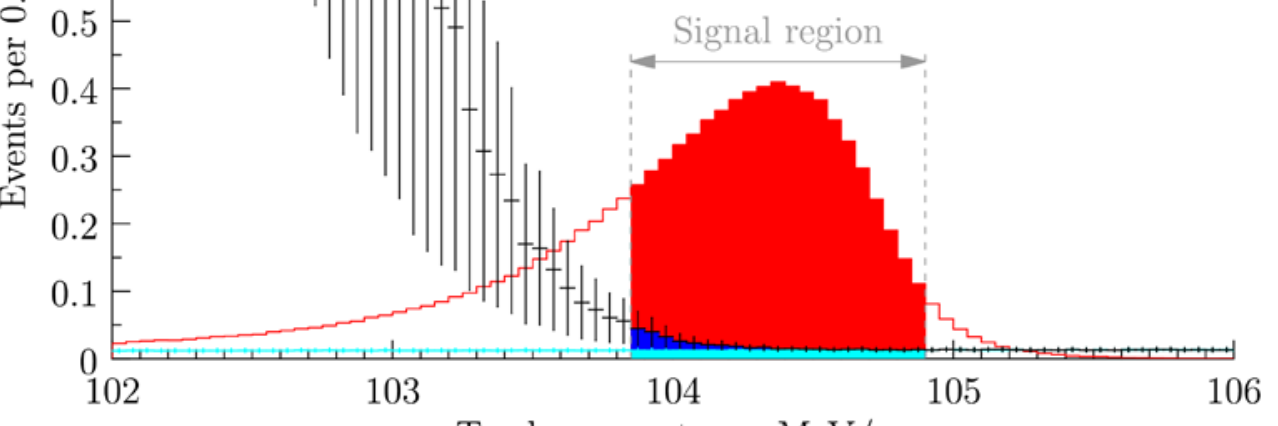

\section{Bottom line:}

Almost background free: Single event sensitivity: background $<0.5$ event

$\mathrm{R}_{\mu e}=3 \times 10^{-17}$ 


\section{COMET experiment - Phase I}

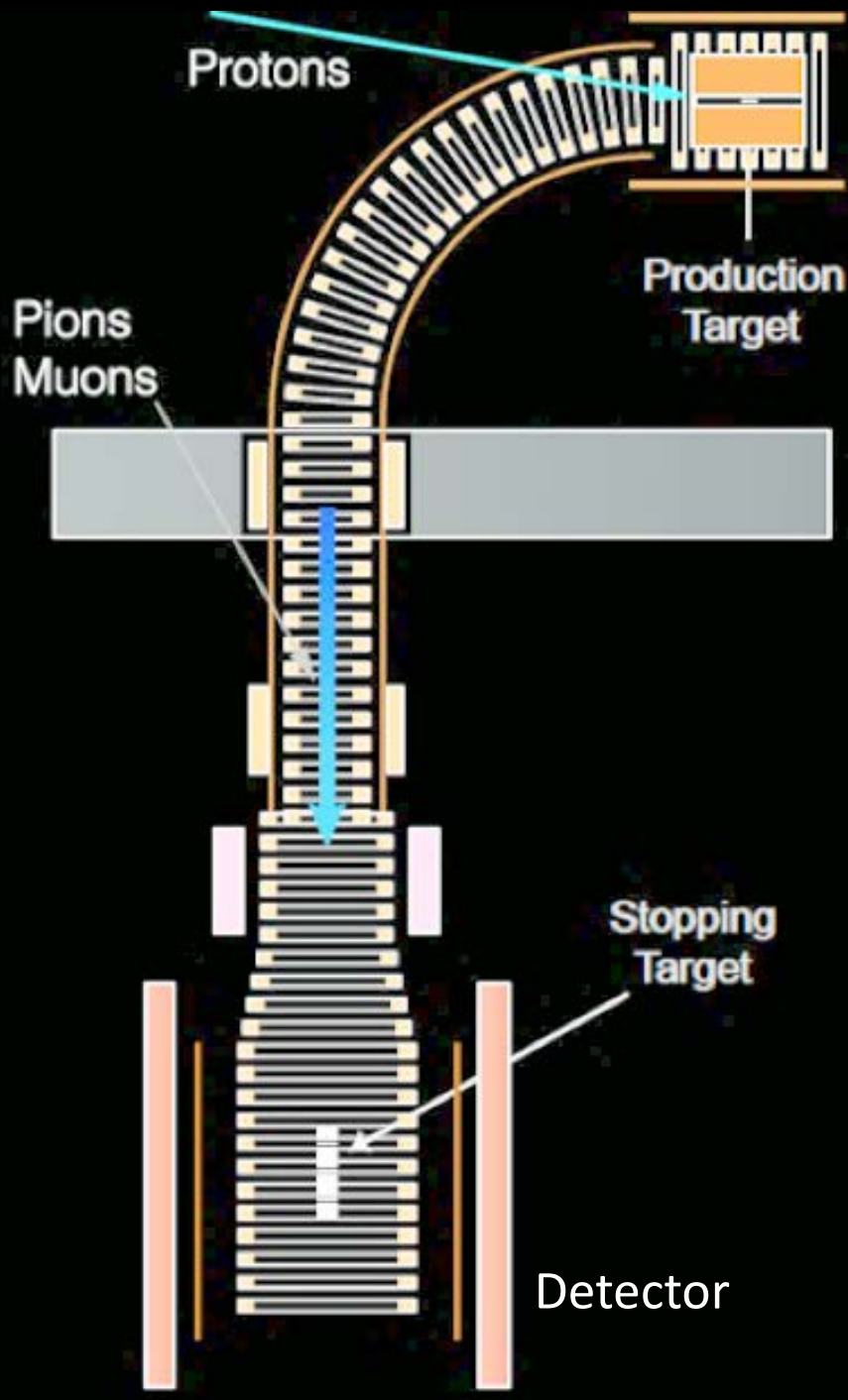

\section{Production target}

Proton beam produces pions by striking target

Pions captured by high solenoidal magnetic field

Transport section

Pion decays to muon

Momentum and charge selection

Stopping target and detector

Muon stops into target

Detector to search for mu-to-e conversion 


\section{COMET experiment - Phase I}
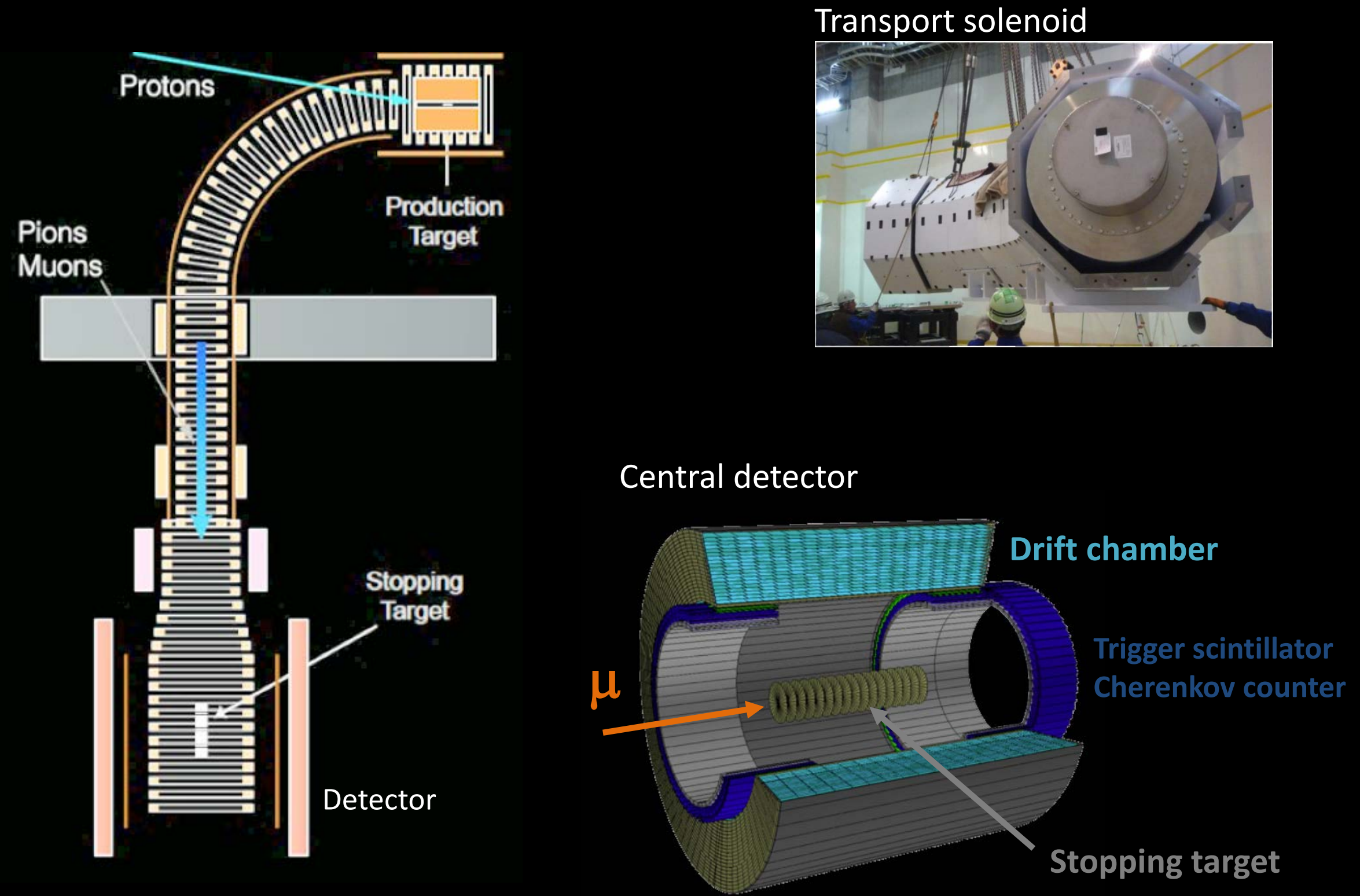


\section{COMET experiment - Phase II}

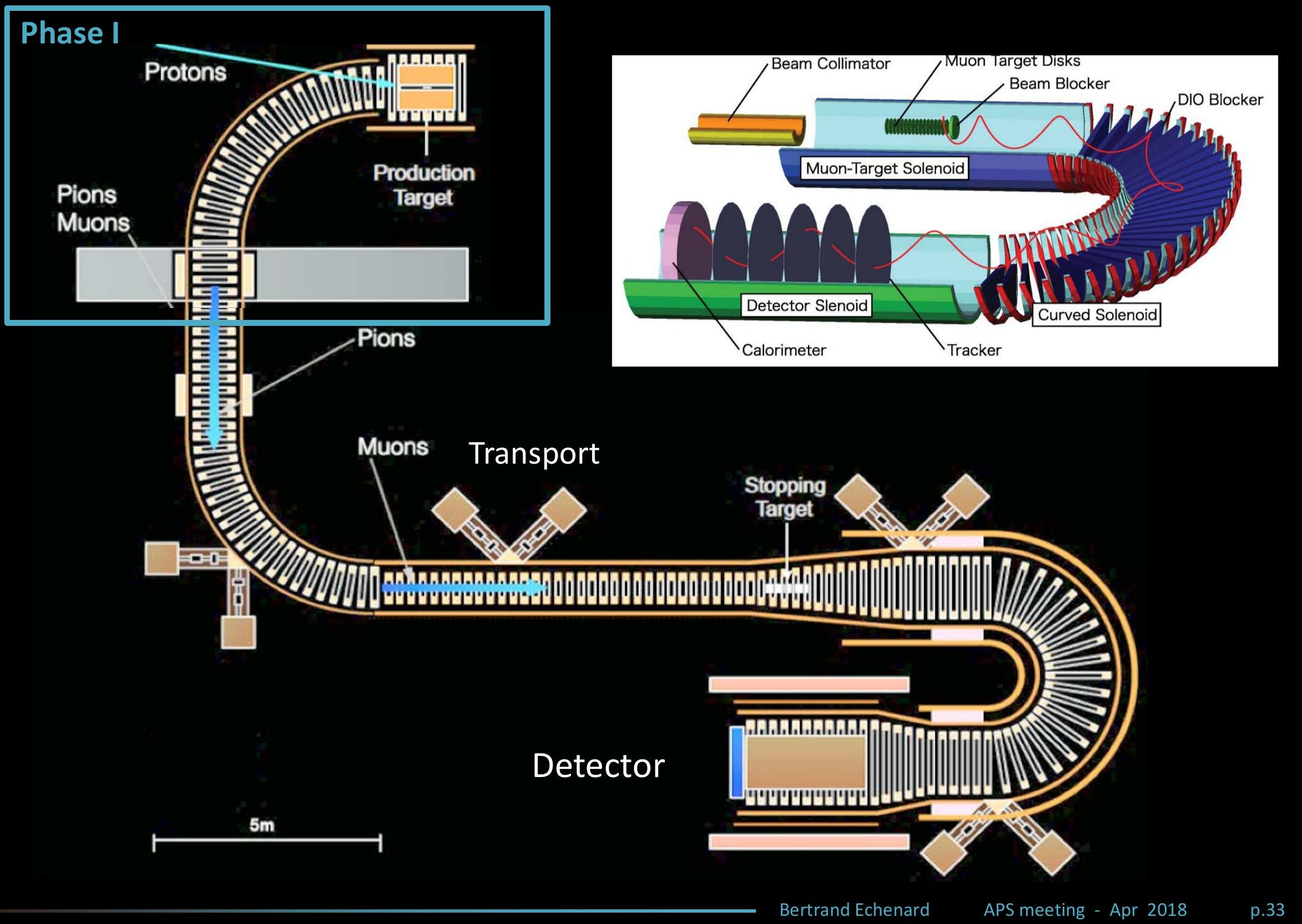




\section{COMET - expected sensitivity}

\section{Estimated sensitivity for Phase I for 150 days of running}

\begin{tabular}{llr}
\hline \hline Type & Background & Estimated events \\
\hline Physics & Muon decay in orbit & 0.01 \\
& Radiative muon capture & 0.0019 \\
& Neutron emission after muon capture & $<0.001$ \\
& Charged particle emission after muon capture & $<0.001$ \\
\hline Prompt Beam & $*$ Beam electrons & \\
& $*$ Muon decay in flight & \\
& $*$ Pion decay in flight & \\
& $*$ Other beam particles & $\leq 0.0038$ \\
& All $(*)$ Combined & 0.0028 \\
& Radiative pion capture & $\sim 10^{-9}$ \\
& Neutrons & $\sim 0$ \\
& Beam electrons & $\sim 0$ \\
& Muon decay in flight & $\sim 0$ \\
& Pion decay in flight & $\sim 0$ \\
& Radiative pion capture & 0.0012 \\
\hline Delayed Beam & $<0.01$ \\
\hline Others & Anti-proton induced backgrounds & 0.032 \\
\hline Total & Cosmic rays & \\
\hline \hline & &
\end{tabular}

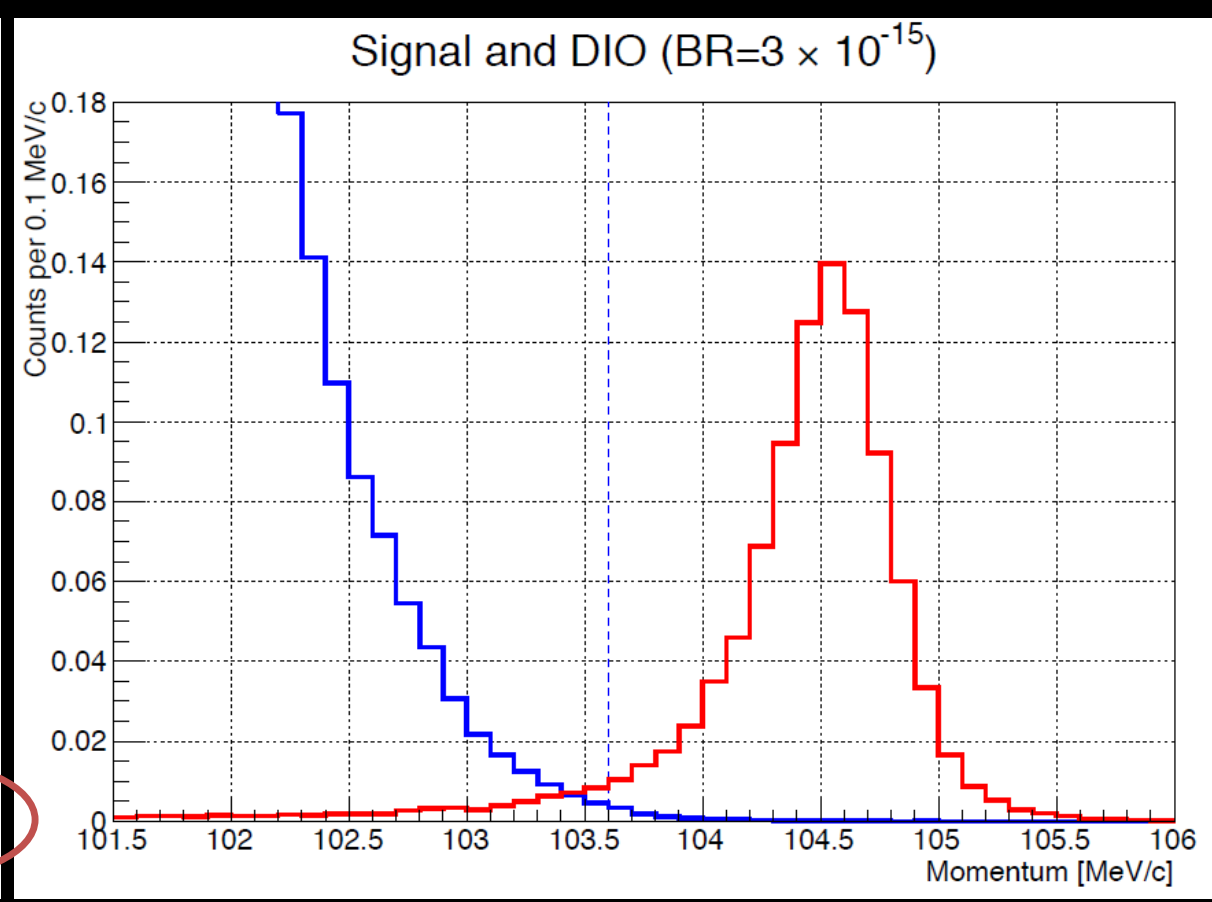

\section{Single event sensitivity:}

Phase I : $R_{\mu e} \sim 3 \times 10^{-15}$ for 150 days of data taking Phase II: $R_{\mu e} \sim 2 \times 10^{-17}$ for $2-3$ years of running 


\section{Schedule}

Mu2e Schedule

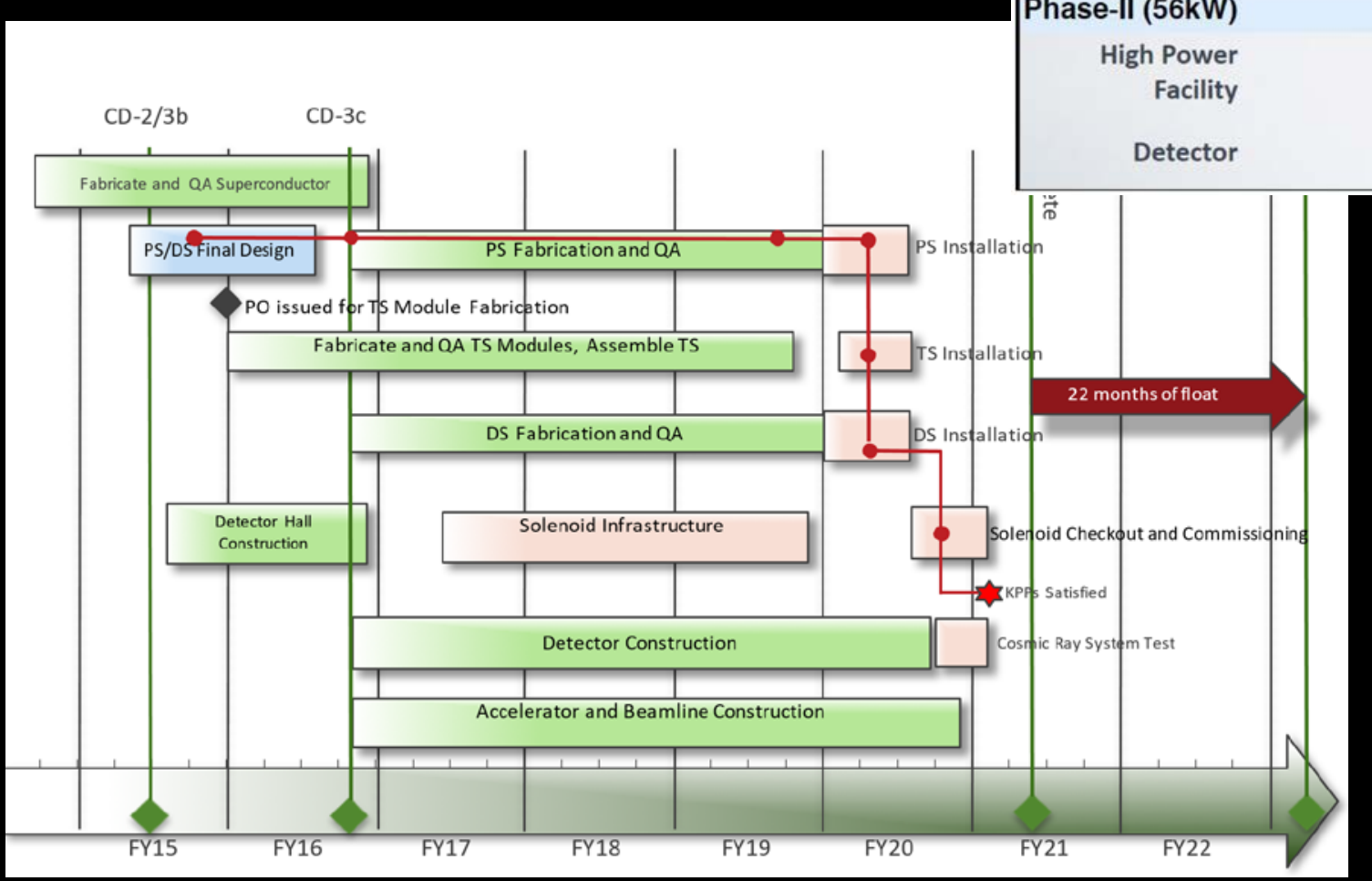

COMET Schedule

\begin{tabular}{|c|c|c|c|c|c|c|c|c|c|c|c|}
\hline & 2015 & 2016 & 2017 & 2018 & 2015 & 2020 & 2021 & 2022 & 2023 & 2024 & 2025 \\
\hline \multicolumn{12}{|l|}{ Phase-I (3.2kW) } \\
\hline Beamline & \multicolumn{11}{|c|}{ High-p/COMET beamline } \\
\hline SC magnet & \multicolumn{11}{|c|}{ Capture, Transport \& Cryogenics } \\
\hline \multirow{2}{*}{ Detector } & \multicolumn{11}{|c|}{ Physics Detector } \\
\hline & \multicolumn{7}{|c|}{ Beam measurement Detector } & \multicolumn{3}{|c|}{$\begin{array}{l}\text { World best sensitivity } \\
\text { measurement }<10^{-14}\end{array}$} & \\
\hline \multicolumn{12}{|l|}{ Data-taking } \\
\hline \multicolumn{12}{|l|}{ Phase-II (56kW) } \\
\hline \multirow{3}{*}{$\begin{array}{r}\text { High Power } \\
\text { Facility }\end{array}$} & & & \multicolumn{9}{|c|}{ Shield, Dump \& Magnet } \\
\hline & & & & & & & xtens & ion & \multirow{2}{*}{\multicolumn{3}{|c|}{$\begin{array}{l}\text { Start measurement toward } \\
\text { the target sensitivity }<10^{-16}\end{array}$}} \\
\hline & & & & & \multicolumn{4}{|c|}{ Phase-II Detector } & & & \\
\hline
\end{tabular}

\section{COMET and Mu2e will start taking data in the coming years}




\section{What's next}

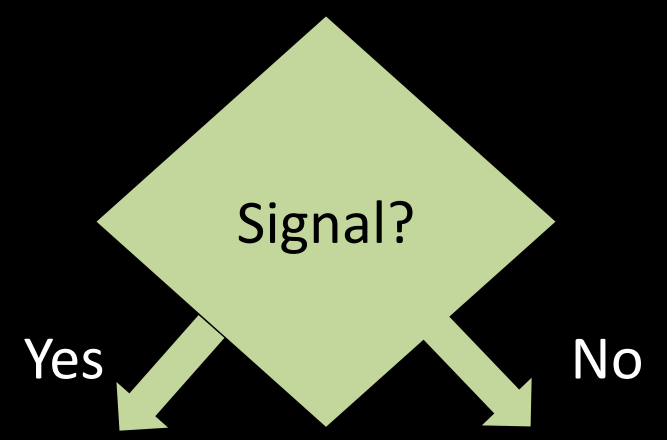

\section{Precision}

measurement

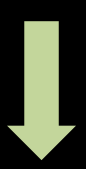

Measure $\mathrm{R}_{\mu e}$ for different targets
$Z$ dependence of $\mu$-to-e conversion

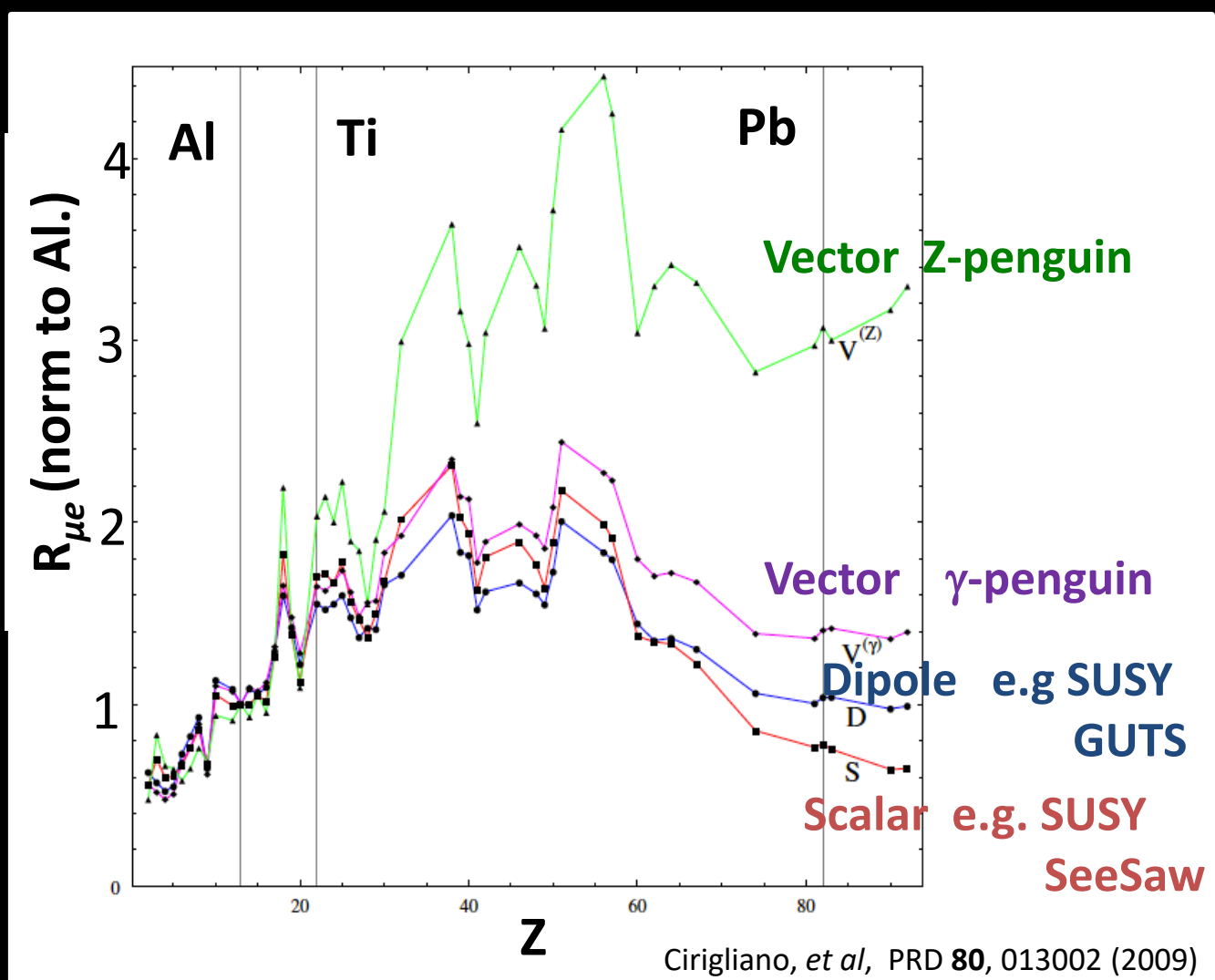

Study underlying BSM physics with different targets if signal is seen 
PIP2 is a superconducting linac for LBNF and the muon campus at FNAL

- $800 \mathrm{MeV}, 100 \mathrm{~s} \mathrm{~kW}$ available for muon campus

- Currently under design

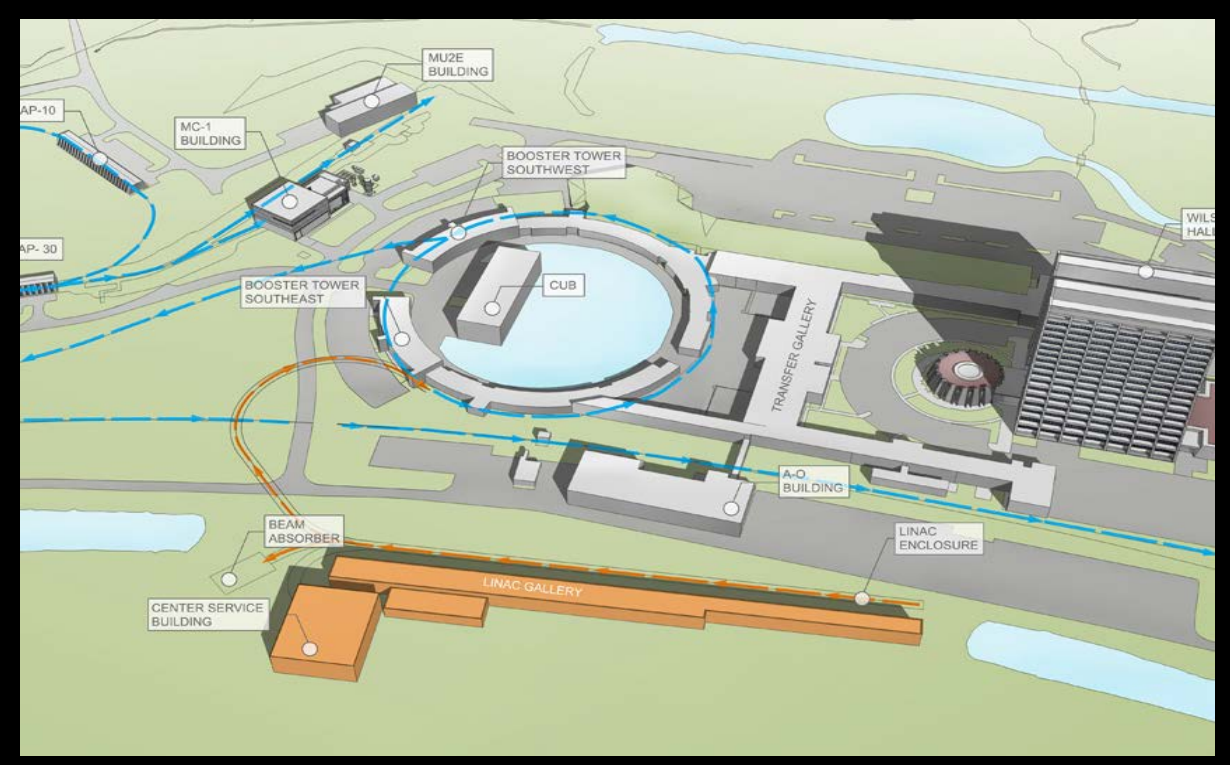

Mu2e II, active study of Mu2e upgrade

- Order of magnitude increase in muon stops with a modest increase in instantaneous rate

- Beamline and detector upgrade

- Improve sensitivity by an order of magnitude

- Presented to Fermilab PAC 2018 
Phase Rotated Intense Slow Muon source PRISM Muon Electron conversion

A muon storage ring (FFAG), feeding a COMET-like detector

Very high muon intensity $10^{11}-10^{12} \mu-/ s$

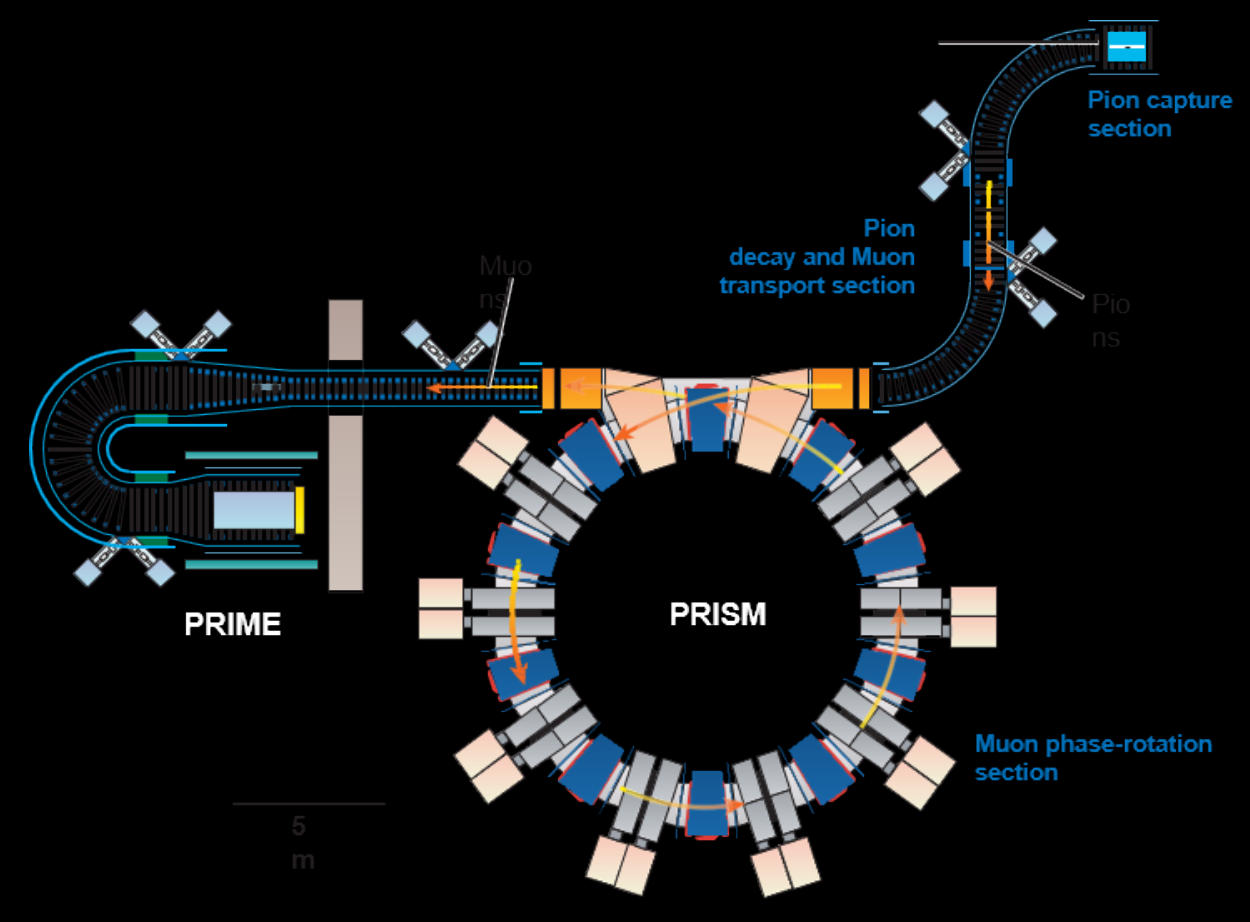

Low momentum, quasi-mono-energetic muons

Timescale $>2030$

Very low pion contamination $<10^{-18}$

Aims for a single event sensitivity at the level of $10^{-19}$ 
CLFV processes:

Generic and powerful probes of BSM physics, touching on the question of flavor and families.

Reach mass scales far beyond the direct production of new particles at the current colliders.

Existing searches already provide useful constraints, and new experiments will explore the regime predicted by many BSM physics.

Analysis of correlations between measurements provide model diagnosis.

Tremendous improvements in several channels is expected over the next decade, e.g. $\mu$-e conversion sensitivity increased by four orders of magnitude. 
CLFV processes:

Generic and powerful probes of BSM physics, touching on the question of flavor and families.

Reach mass scales far beyond the direct production of new particles at the current colliders.

Existing searches already provide useful constraints, and new experiments will explore the regime predicted by many BSM physics.

Analysis of correlations between measurements provide model diagnosis.

Tremendous improvements in several channels is expected over the next decade, e.g. $\mu$-e conversion sensitivity increased by four orders of magnitude 


\section{EXTRA MATERIAL}




\section{Higgs mediated CLFV decays}

Bounds on CLFV couplings to the Higgs are derived from many measurements

$\mathcal{L}_{Y} \supset-Y_{e \mu} \bar{e}_{L} \mu_{R} h-Y_{\mu e} \bar{\mu}_{L} e_{R} h-Y_{e \tau} \bar{e}_{L} \tau_{R} h-Y_{\tau e} \bar{\tau}_{L} e_{R} h-Y_{\mu \tau} \bar{\mu}_{L} \tau_{R} h-Y_{\tau \mu} \bar{\tau}_{L} \mu_{R} h+$ h.c..

CLFV Higgs decay $\quad \Gamma\left(h \rightarrow \ell^{\alpha} \ell^{\beta}\right)=\frac{m_{h}}{8 \pi}\left(\left|Y_{\ell^{\beta} \ell^{\alpha}}\right|^{2}+\left|Y_{\ell^{\alpha} \ell^{\beta}}\right|^{2}\right)$
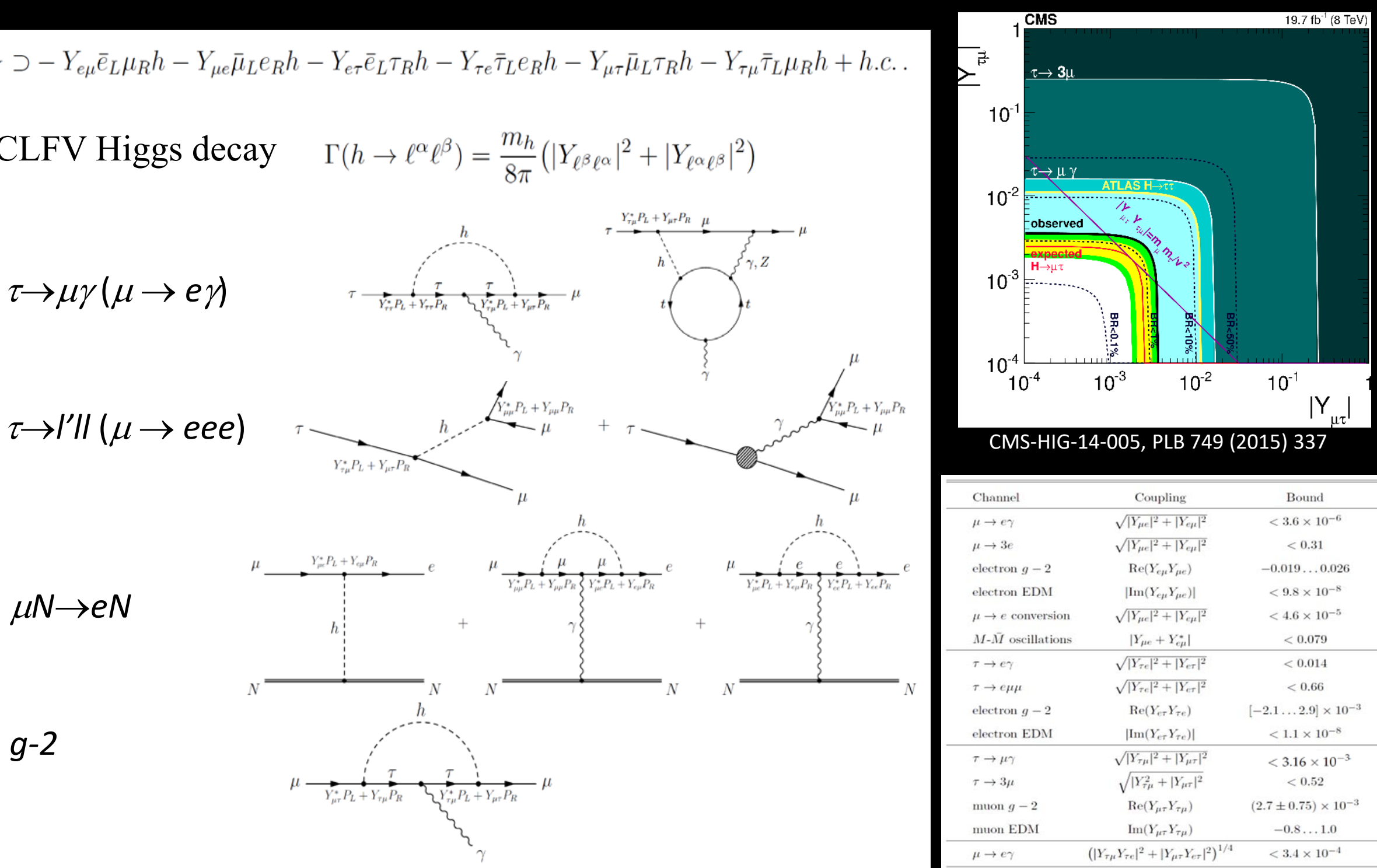

CMS-HIG-14-005, PLB 749 (2015) 337

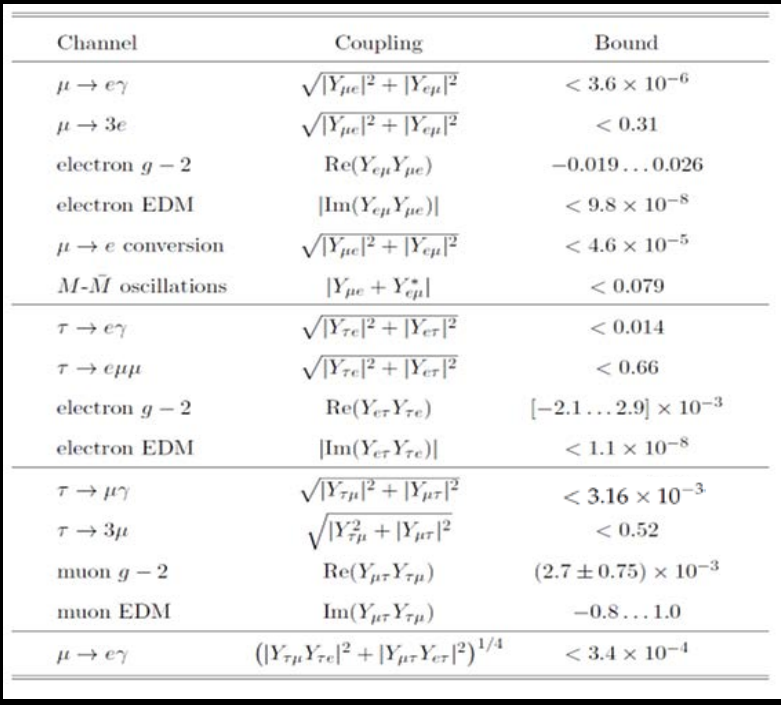




\section{CLFV physics reach}

$$
\mathcal{L}_{\mathrm{CLFV}}=\frac{m_{m}}{(1+k) \Lambda^{2}} \bar{m}_{R} s_{m} e_{L} F^{m}+\frac{k}{(1+k) \Lambda^{2}} \bar{m}_{L} g_{m} e_{L}\left(\bar{e}_{L} g_{m} e_{L}\right)+\text { h.c. }
$$
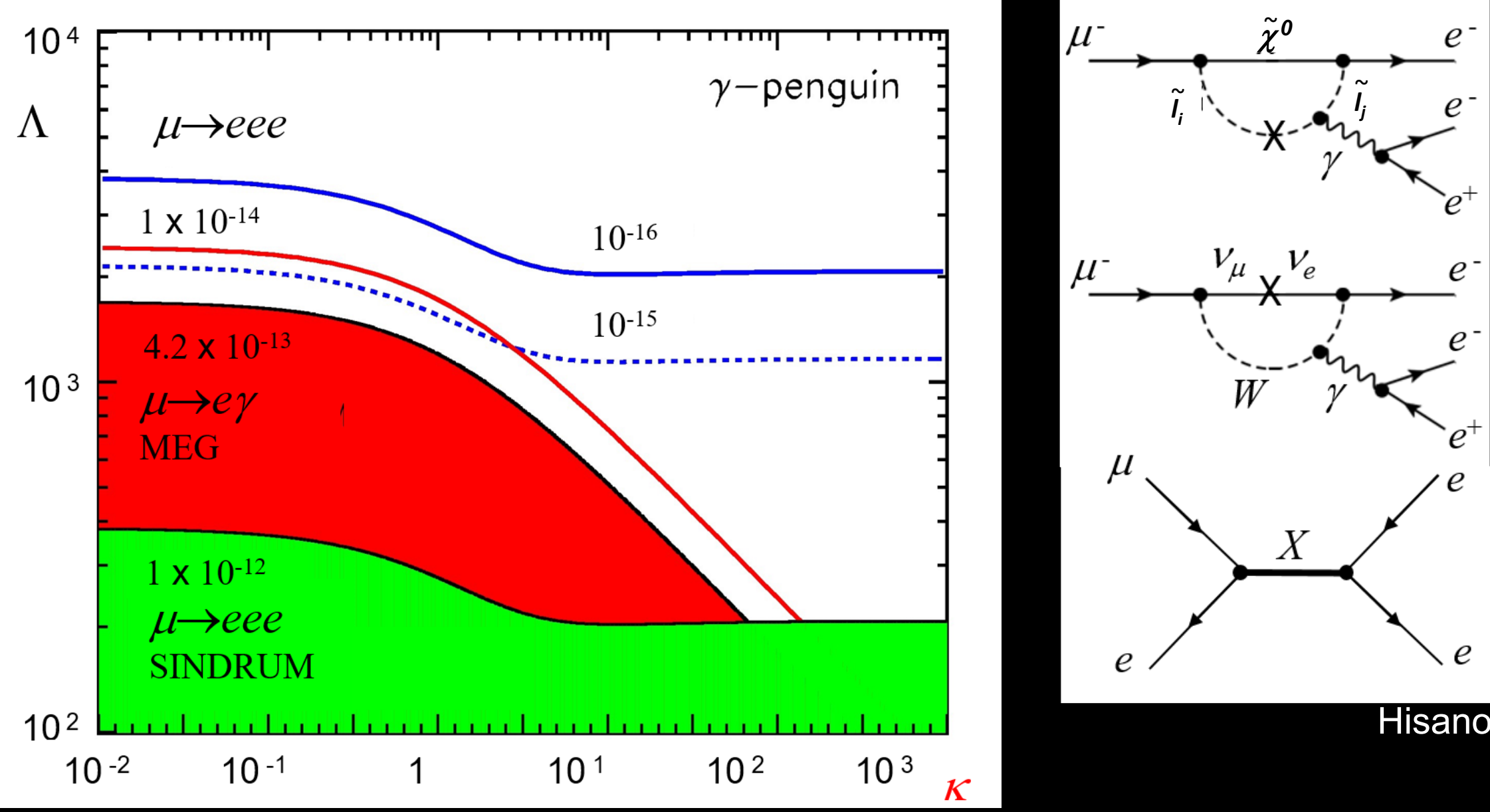

Hisano 


\section{CLFV tau decays}

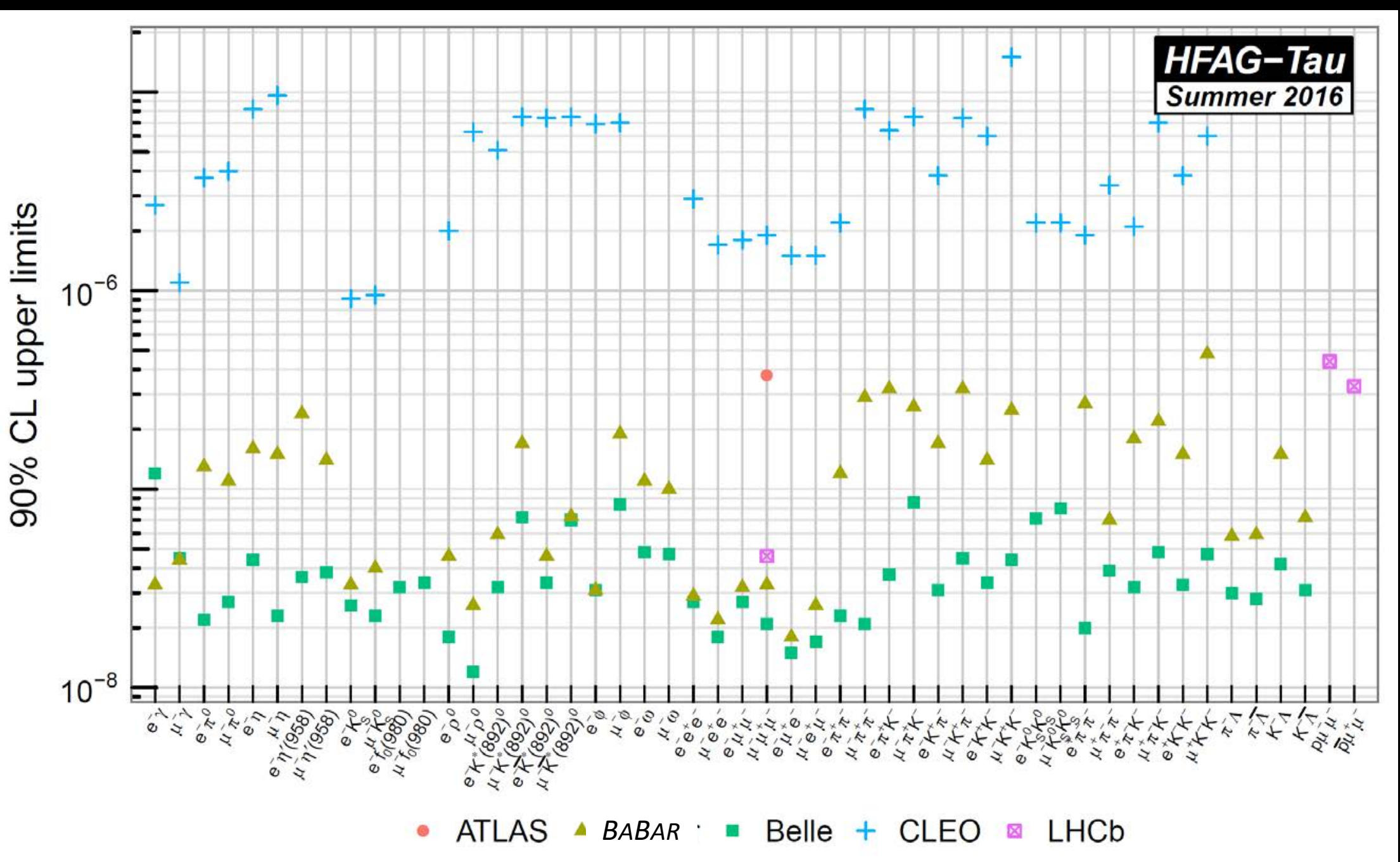




\section{Current best limits - SINDRUM II at PSI}

W. Bertl et al., Eur. Phys. J. C 47, 337-346 (2006)

SINDRUM II at PSI

Final results on Au:

$$
R_{\mu e}<7 \times 10^{-13} @ 90 \% \mathrm{CL}
$$

Timing cut shows the contribution of prompt background (0.3 ns muon pulse separated by $20 \mathrm{~ns}$ )

One candidate event past the end of the spectrum. Pion capture, cosmic ray?

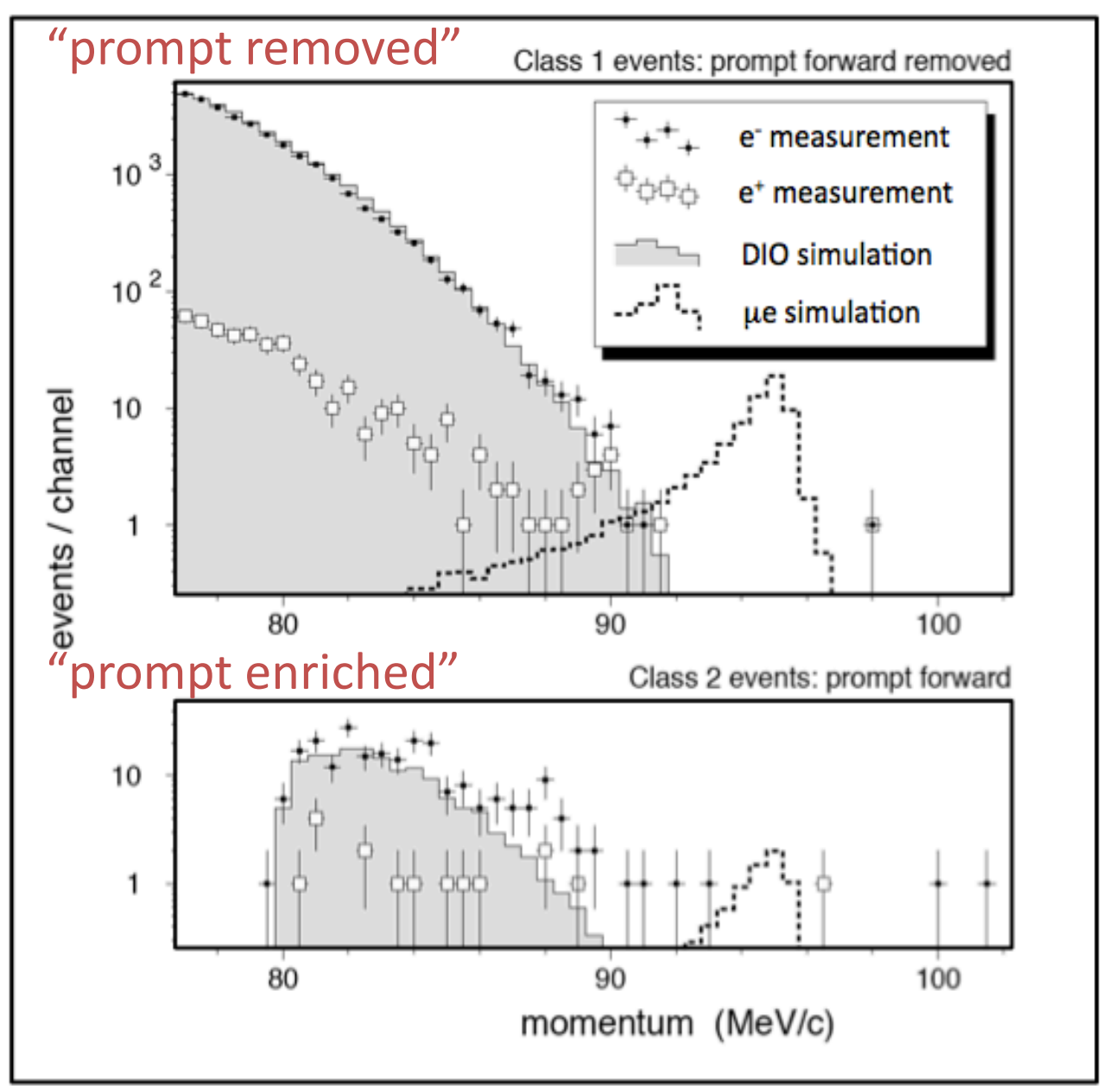




\section{Mu2e experiment - inside a microbunch}

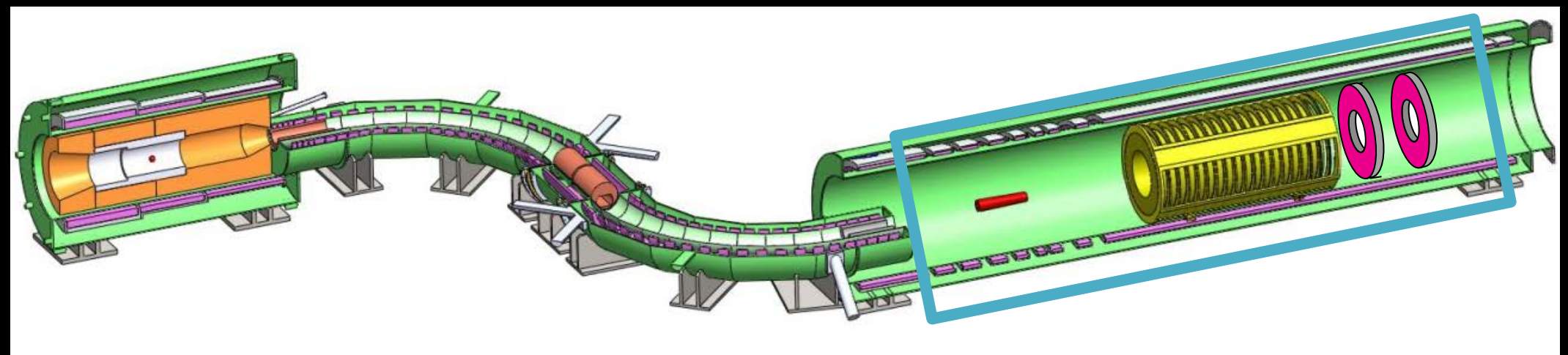

Particles produced in an event, integrated over a window of $500-1695$ ns after the protons hit the primary target

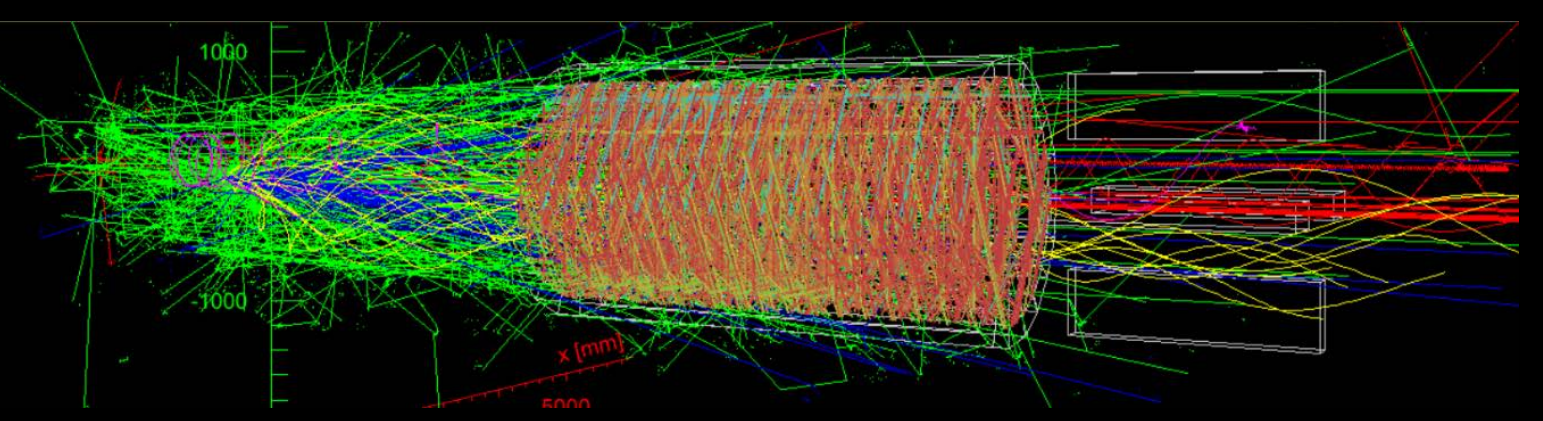

A window of \pm 50 ns around a potential signal candidate

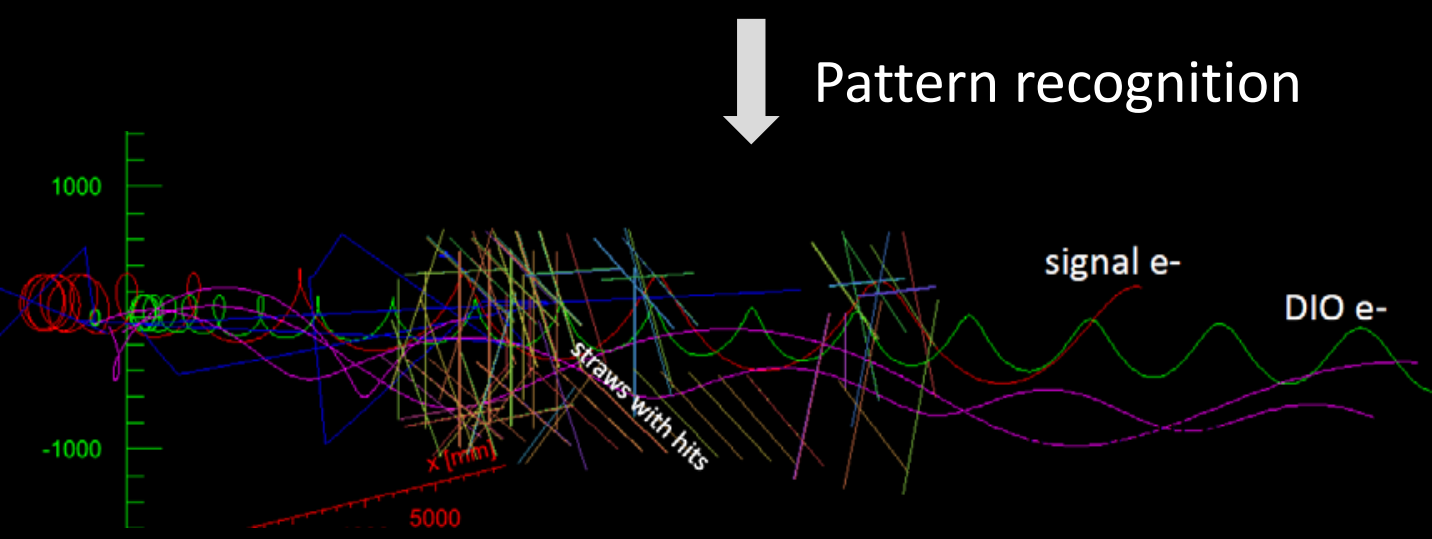


Fermilab ideally suited for Mu2e

Booster: batch of $4 \times 10^{12}$ protons every $1 / 15^{\text {th }}$ second

Booster "batch" is injected into the Recycler ring and rebunched into 4 bunches

These are extracted one at a time to the Debuncher ring

Protons are extracted into bunches of $\sim 3 \times 10^{7}$ protons each, separated by $1.7 \mu \mathrm{s}$ (debuncher ring period)

Achieve an extinction: \#protons out of bunches/\#proton in bunch $\sim 10^{-10}$ or better

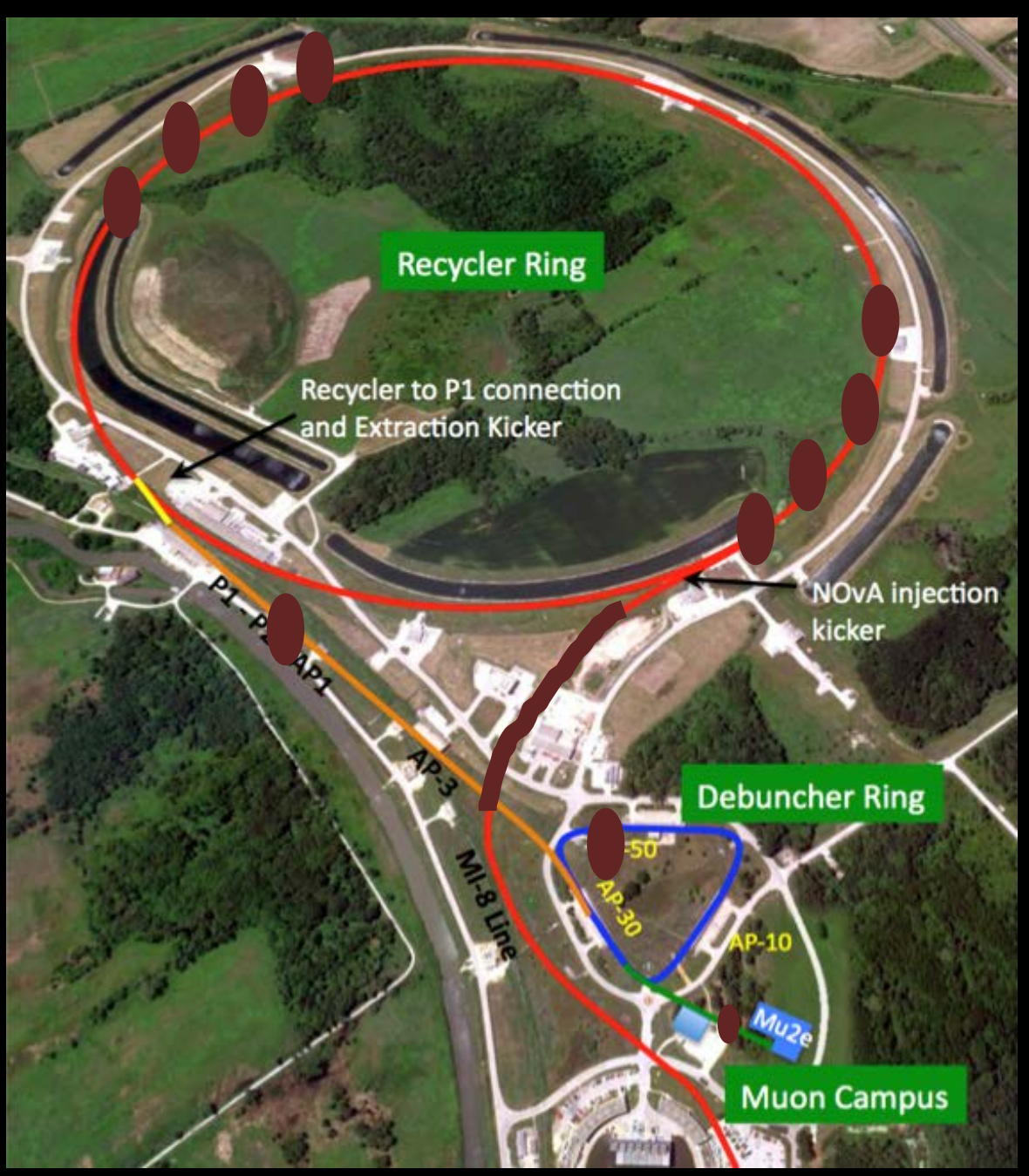

Pulse structure: width $\ll \tau_{\mu}(\mathrm{Al})$ with separation $\sim 2 \mathrm{x} \tau_{\mu}(\mathrm{Al})$ 


\section{DeeMee}

Search for $\mu \rightarrow e$ conversion with a high power, high purity proton beam at J-PARC

Conversion happens in production target. Initially graphite target, upgrade to SiC
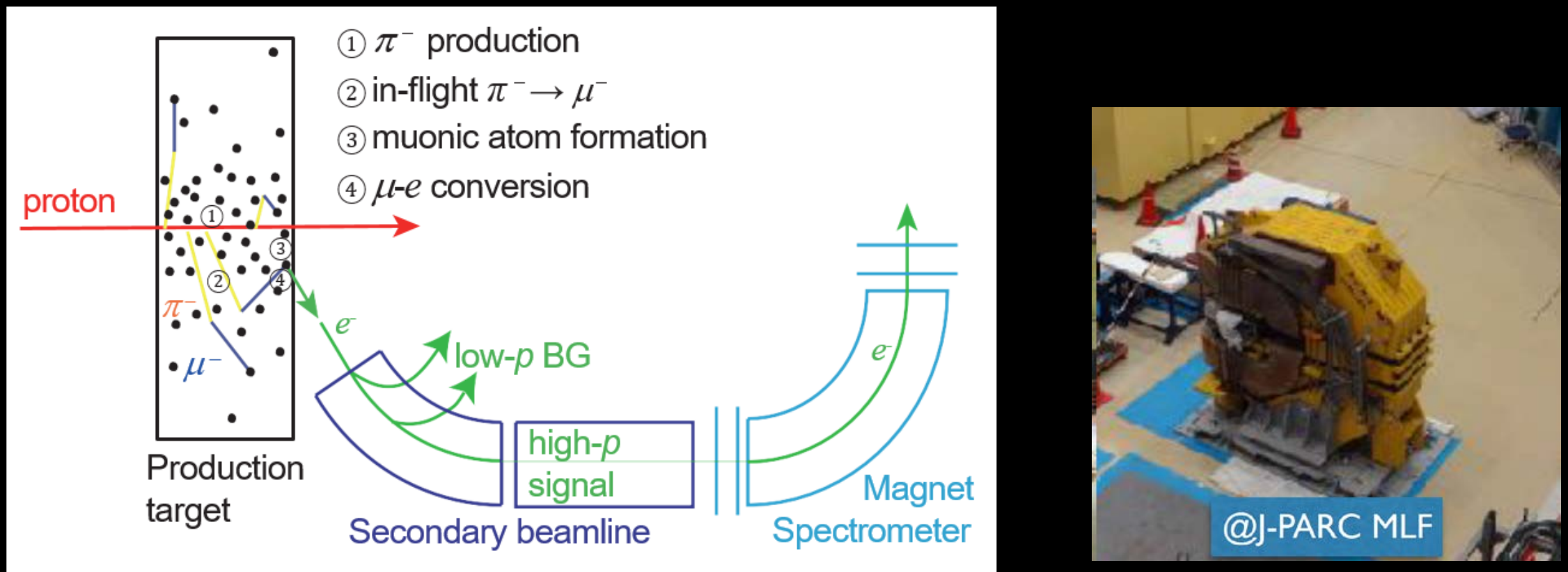

Single event sensitivity with $1 \mathrm{MW}$ beam :

1 year: $\quad 1.2 \times 10^{-13} \rightarrow 2.1 \times 10^{-14}$ upgrade

4 years: $2.5 \times 10^{-14} \rightarrow 5 \times 10^{-15}$ upgrade 